\title{
INS Assisted Fuzzy Tracking Loop for GPS-Guided Missiles and Vehicular Applications
}

\author{
Ahmed M. Kamel, ${ }^{1,2}$ Valerie Renaudin,,3 John Nielsen, ${ }^{1}$ and Gérard Lachapelle ${ }^{1}$ \\ ${ }^{1}$ Position Location and Navigation (PLAN) Group, Schulich School of Engineering, University of Calgary, Calgary, AB, \\ Canada T2N 1 N4 \\ ${ }^{2}$ Egyptian Armed Forces, Military Technical College, Kobry Elkobbah, Cairo, Egypt \\ ${ }^{3}$ GEOLOC Group, Department of Components and Systems, IFSTTAR, 44344 Bouguenais, France
}

Correspondence should be addressed to Valerie Renaudin; valerie.renaudin@ifsttar.fr

Received 24 July 2012; Accepted 14 December 2012

Academic Editor: Yuei-An Liou

Copyright (C) 2013 Ahmed M. Kamel et al. This is an open access article distributed under the Creative Commons Attribution License, which permits unrestricted use, distribution, and reproduction in any medium, provided the original work is properly cited.

\begin{abstract}
Autonomous Navigation Systems used in missiles and other high dynamic platforms are mostly dependent on the Global Positioning System (GPS). GPS users face limitations in terms of missile high dynamics and signal interference. Receiver's tracking loops bandwidth requirements to avoid these problems are conflicting. The paper presents a novel signal frequency and phase tracking algorithm for very high dynamic conditions, which mitigates the conflicting choice of bandwidths and reduces tracking loop measurement noise. It exploits the flexibility of fuzzy control systems for directly generating the required Numerically Controlled Oscillator (NCO) tuning frequency using phase and frequency discriminators information and is labeled Fuzzy Frequency Phase Lock Loop (FFPLL). Because Fuzzy Systems can be computationally demanding and an Inertial Navigation System (INS) is often onboard the vehicle, an assisted INS Doppler version has been designed and is also proposed. Assessment of the new GPS tracking method is performed with both simulated and experimental data under jamming conditions. The main enhancements of the proposed system consist in reduced processing time, improved tracking continuity and faster reacquisition time.
\end{abstract}

\section{Introduction}

It is often desirable to track carrier phase information for GPS-based applications that require a high level of measurement accuracy even if carrier phase tracking is more difficult than tracking the frequency. A Phase Lock Loop (PLL) can be used to track the incoming GPS carrier phase and hence extract the modulated navigation data. If the GPS receiver is expected to perform at high levels of dynamics, the PLL can be aided by a Frequency Lock Loop (FLL) as a FLL-assisted PLL structure [1]. Figure 1 [2] shows the basic block diagram of a standard FLL assisted PLL. The two first multiplication stages are required to wipe off the input signal carrier and Pseudo-Random Noise (PRN) code required for any Code Division Multiple Access (CDMA) communication system. A local replica of the PRN code is provided by the Delay Lock Loop (DLL) and is used to remove the PRN sequence from the incoming signal. The frequency and phase loop discriminators are used to estimate the frequency and phase error, respectively, between local and incoming carriers. The discriminator's outputs, which represent the frequency and phase errors, are then filtered and used to tune the Numerically Controlled Oscillator (NCO), which adjusts the frequency of the local carrier wave. In this way, the local carrier wave tends to be a precise replica of the input signal carrier. FLL-aided PLL is a typical GPS tracking loop design.

PLLs are required to have the narrowest bandwidth (BW) possible to reduce the impact of signal noise and interference [3]. A conflicting requirement is that the loop bandwidth has to be as wide as possible to accommodate high dynamics. An FLL assisted PLL structure can help to increase the dynamics threshold level to accomplish this. But this threshold will not be sufficient to accommodate high levels of dynamics 
especially if a narrow bandwidth is used, and for that reason different approaches are usually used in the context of GPSguided munitions such as external aiding and Kalman filter(KF-) based tracking loops [4].

Kalman filter-based PLLs constitute a practical solution commonly used for GPS signal tracking [5]. However, it is very hard to correctly tune the measurements noise parameters in the presence of unexpected interference. Indeed, a correctly tuned KF provides a better tracking performance than standard PLLs in normal cases, but unexpected external disturbances can lead to divergence of the filter, and loss of phase lock can occur [6].

The tracking limitation of the abovementioned algorithms motivated the design of a new tracking algorithm that is capable of maintaining signal tracking in high dynamics conditions and is also able of coping with signal interference and providing measurements with reduced noise. This novel algorithm depends on fuzzy Logic theory [7] and was first introduced in [8].

Fuzzy Logic-based Phase Lock Loop algorithms have shown better performance in terms of dynamic robustness and associated with Doppler noise as compared with a standard PLL $[8,9]$. A main drawback in the performance of the Fuzzy based tracking system is its computational load. Taking advantage of onboard inertial sensors that are commonly or becoming available in missiles and other platforms, system performance can be further improved using inertial aiding to provide frequency information. The number of fuzzy inputs is then reduced to only one corresponding to the phase tuning input, which is defined by the frequency's change required for cancelling the phase shift. Consequently, the computational load of the Fuzzy Logic-based PLL is drastically diminished. Therefore a novel inertial-assisted Fuzzy Logic-based Frequency Lock Loop is presented herein.

The coupling between GPS and an inertial navigation system (INS) [10] not only provides a good solution for high-rate manoeuvring platforms, but also provides a good and reliable solution for the interference problem, that is, when typical tracking loop's designs fail due to the presence of an external signal perturbation's source. Indeed INSs are not affected by external interference. First, GPS can be used to periodically calibrate the biases in the INS. Second, INS measurements can assist GPS based navigation to improve acquisition, track times, and handle multipath at launch. Classically GPS and INS are coupled via Kalman techniques [6] to fuse navigation information and handle short intervals of GPS outage caused by interference or jamming. The levels of achieved performance depend on INS accuracy, that is, the grade level of the inertial sensors and GPS satellite visibility. The tradeoff is generally driven by miss distance requirements. The complexity and cost of coupling are generally acceptable for missiles to carry both systems. Furthermore for missile systems, coupling becomes necessary to handle the extreme dynamics, rapidly changing GPS visibility, and jamming. Another mathematical tool that suits coupling of INS and GPS signals is Fuzzy Logic. It has already been used in some research for enhancing the performance of an integrated GPS/INS systems but, to the authors' knowledge, existing research has not focused on improving the tracking loop in the context of interference mitigation $[11,12]$.

The advantages of GPS/INS integration, relative to either GPS or INS alone, can be summarized as follows $[13,14]$ :

(i) a high data rate of complete navigation solutions, including position velocity and attitude,

(ii) superior short-term and long-term positioning accuracies,

(iii) continuous availability,

(iv) smoother trajectories,

(v) greater integrity, and

(vi) nonsignificant cost specially when used for military applications.

Depending on the data fusion strategy, it is possible to sort integrated GPS/INS systems into three different types: loosely coupled GPS/INS, tightly coupled GPS/INS, and ultratightly coupled GPS/INS. The ultratightly coupled integration technique [15-17], used herein, combines the INS/GPS solution and the GPS signal tracking functions into a single estimation algorithm in which the INS measurements are fed back to the receiver to decrease GPS signal tracking errors and enhance GPS positioning performance. This scheme also helps the receiver tracking loop for retrieving signal lock if it is lost due to interference or jamming. A block diagram illustration of an ultratightly coupled integration strategy is shown in Figure 2.

After describing the original design of the fuzzy frequency Phase Lock Loop in Sections 2 and 3 presents the necessary modifications needed for integrating inertial measurements. Section 4 describes the experimental test. Finally the results and associated conclusions are presented. It is shown that the main enhancements of the INS-assisted Fuzzy based tracking loop consist in a reduction of processing time, better tracking continuity, and faster reacquisition time, characteristics that are of fundamental importance for the most high dynamics applications.

\section{Fuzzy Frequency Phase Lock Loops}

Automatic control methods based on artificial intelligence (e.g., Fuzzy Systems, Neural Networks, and Genetic Algorithms) have emerged as an alternative model to analytic control theory [18]. One of the greatest advantages of fuzzy controllers is the simple and intuitive design, which is usually based on the designer experience and experiments. However, this advantage is sometimes considered a drawback too. Indeed this simplicity and the lack of theoretical performance justifications are perhaps the primary cause of their initial slow acceptance among the control community [7].

The mathematical basis of fuzzy inference/implication enables fuzzy models to provide similar discrete or deterministic results as the ones obtained from conventional knowledge-based systems [19]. The operations of transforming discrete values to linguistic variables and vice versa are called Fuzzification and Defuzzification, respectively. In Fuzzy Logic applications, linguistic variables are nonnumerical elements that are used to enable the expression of 


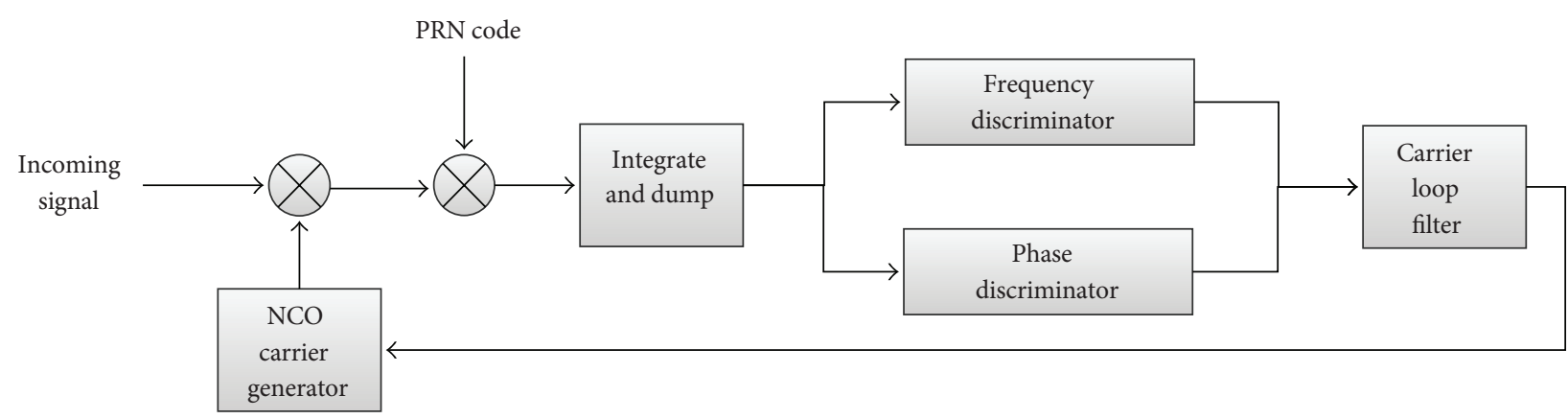

FIGURE 1: FLL-assisted PLL structure.

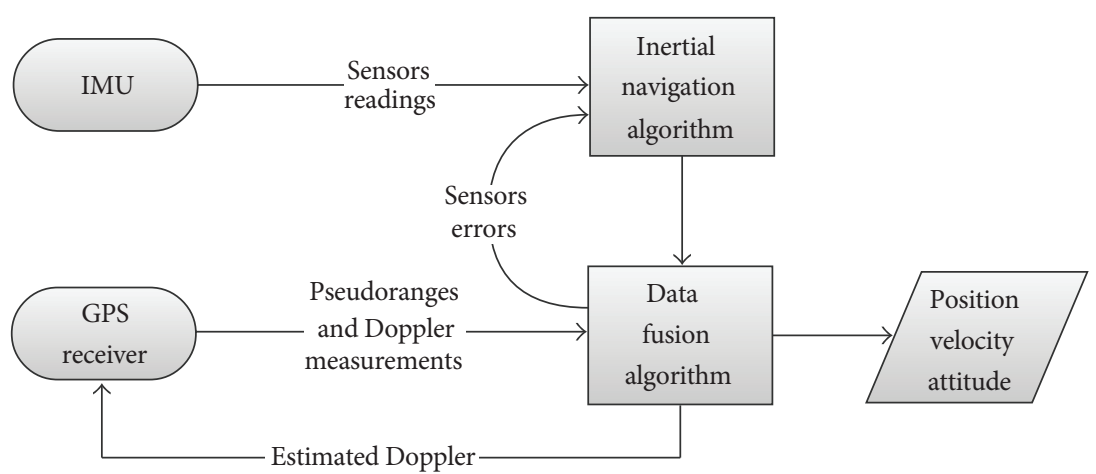

FIGURE 2: Block diagram of an ultratightly coupled GPS/INS integration algorithm.

rules and facts. Using a priori knowledge of the problem, the fuzzy system designer is able to choose the appropriate membership functions and construct sufficient rules to fulfill the correct filtering operation. A schematic illustration of a fuzzy inference process is shown in Figure 3. The specific design of the proposed FFPLL is now described.

The basic structure of the proposed Fuzzy based tracking system design was first presented in [8]. In this scheme, the standard loop FLL assisted PLL filter is replaced by the proposed Fuzzy Frequency Phase Lock Loop (FFPLL) controller, which accepts the phase and frequency discriminator output signals as inputs, as illustrated in Figure 4. Indeed, the FFPLL design comprises two input fuzzy variables and one control fuzzy variable. The first state variable is the phase error from the phase discriminator output. The second state variable is the frequency error from the frequency discriminator output. The control fuzzy variable is the required tuning frequency of the NCO to generate an exact replica of the incoming signal. The Fuzzy controller is composed of three consecutive layers, namely, Fuzzification, Fuzzy Associative Memories (FAMs), which are also called "Fuzzy rules" or associations, and Defuzzification layers. A Fuzzification layer is composed of a number of fuzzy sets characterized by Membership Functions (MFs). In this work these MFs are chosen to follow the Gaussian distribution whose parameters depend on the signal quality, as described explicitly in [20] and now briefly recalled. These MFs are responsible for converting the crisp input values into linguistic values. The Defuzzification layer is related to the Fuzzification layer through the FAM rules, which composes the second layer. Each input has a Fuzzification stage composed of nine different fuzzy sets. The total number of fuzzy rule combinations is therefore 9 by 9 , hence 81 rules that are all processed in parallel at different degrees. Each FAM translates a fuzzy set into variables that have been determined in a learning phase. It represents ambiguous expert knowledge or learned inputoutput transformations. The system nonlinearly transforms exact or fuzzy state inputs to a fuzzy set output. This output is defuzzified with a centroid operation [19] to generate an exact numerical output, which gives the NCO tuning signal.

Although it has been shown in [8] that the FFPLL provides better performance than standard tracking using PLL or FLL/PLL in challenging conditions, a main concern remains, namely, the reduction of processing time required to solve for all fuzzy rules in parallel. Taking advantage of the use of an INS, it was decided to exploit inertial signals to aid the FFPLL in a new form of GPS/INS ultratight integration. The proposed method to exploit an INS for assisting the FFPLL is described in Section 3.

The fuzzy frequency/phase tracking system is designed to rapidly recover the signal frequency in the presence of large frequency errors, that is, after acquisition/reacquisition, and to behave as a PLL, with precise phase recovery, in the case of small frequency errors. The fuzziness of the system inputs is mainly due to the low power of GPS signals with respect to thermal noise, which is the main source of phase/frequency jitter, and to the presence of interference [4]. Noise distribution then plays a major role in the system 


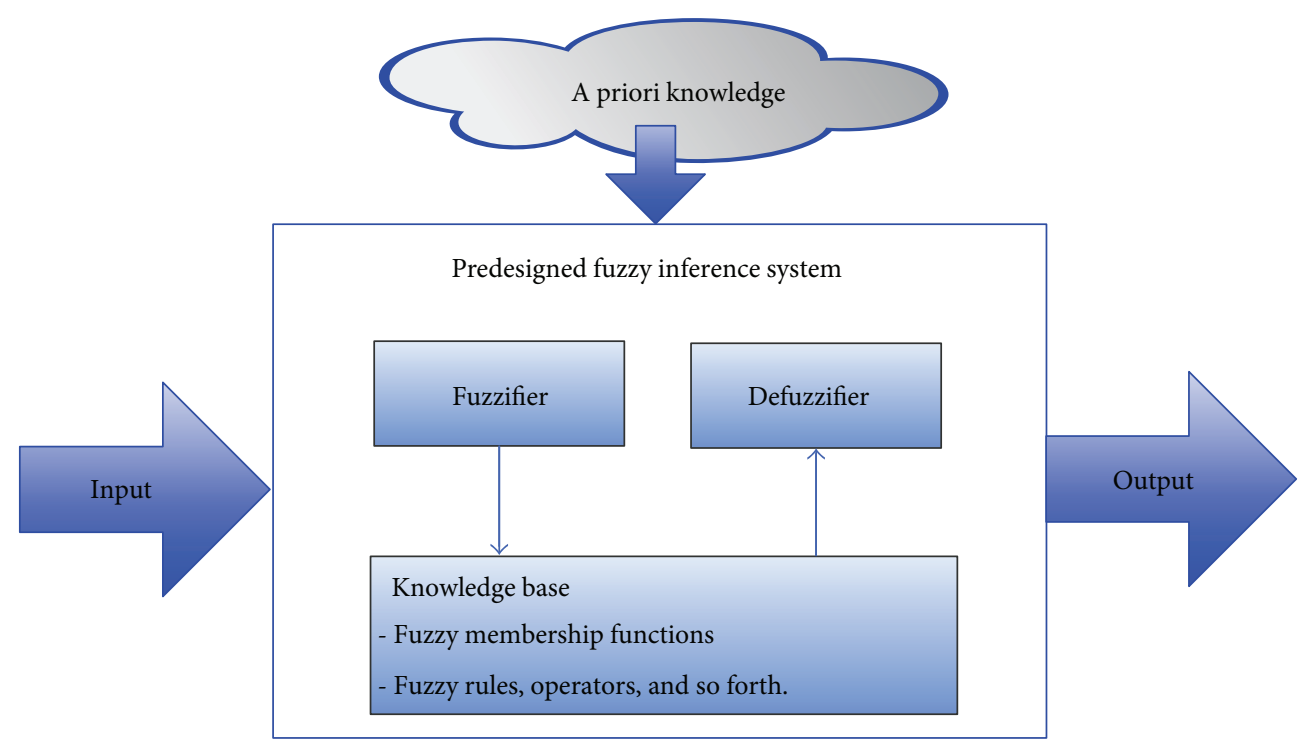

Figure 3: Fuzzy inference process.

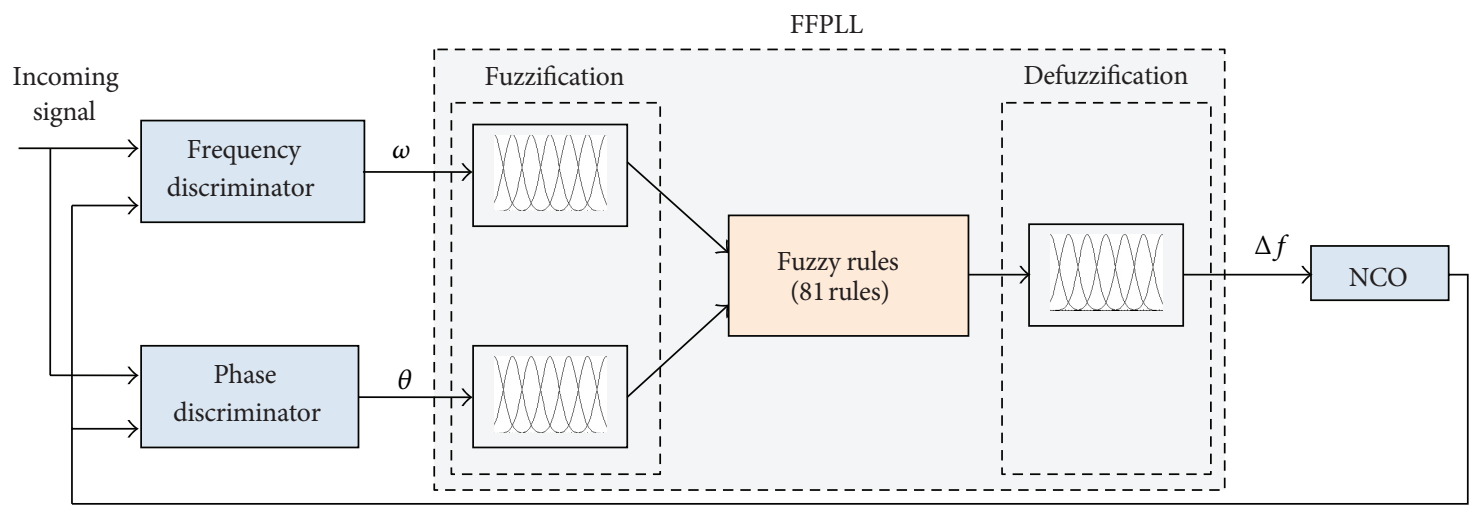

Figure 4: FFPLL original design.

design. This is why an a priori knowledge of expected signal parameters such as $C / N_{0}$ is essential. This knowledge can be achieved during signal acquisition or in the first stages of signal tracking. As shown in Figure 5, a signal with a $C / N_{0}$ of $39 \mathrm{~dB}-\mathrm{Hz}$ in static conditions and in an interference free environment is characterized by a phase discriminator output whose distribution is Gaussian.

Due to the nonlinearity of the arctangent operator used herein, a theoretical expression of the phase error variance is not used. Instead it is assumed that the equation, which is applicable to the dot-product discriminator, also suits the arctangent discriminator [21]. When using a standard PLL, the standard deviation (SD) of the phase discriminator output can be then theoretically calculated from [22] as

$$
\sigma_{\theta}^{2}=\frac{1}{2 c / n_{0} T}\left(1+\frac{1}{2 c / n_{0} T}\right)
$$

where $\sigma_{\theta}^{2}$ is the variance of the dot-product discriminator, $T(\mathrm{~s})$ is the predetection integration time, and $c / n_{0}$ gives carrier to noise power expressed as a ratio $(\mathrm{Hz})$ where $c / n_{0}=10^{\left(C / N_{0}\right) / 10}$ for $C / N_{0}$ expressed in $\mathrm{dB}-\mathrm{Hz}$. The standard deviation for the frequency error is not included in (1) because only the phase discriminator is used in the new INS-assisted FFPLL.

Figure 5 shows the time domain representation of the phase discriminator output during tracking of the incoming signal received from PRN 5 using a $4 \mathrm{~Hz}$ third-order PLL with $1 \mathrm{~ms}$ coherent integration time and its histogram approximated as Gaussian. The corresponding Gaussian Probability Density Function (PDF) covers the signal expected values under standard tracking conditions at a certain $C / N_{0}$ level, and it can be linguistically described as zero state if compared to the ideal phase discriminator output. The mean and standard deviation, which are the two parameters governing the Gaussian distribution function, are directly related to the signal dynamics and signal quality, respectively. Receiver dynamics can cause a phase tracking error, and hence the distribution mean will be shifted from zero. On the other hand, the changes in the signal quality will result in changes in the standard deviation as illustrated in (1). An appropriate mapping between the signal PDF and fuzzy MFs can be 
TABLE 1: Distribution of fuzzy membership functions.

\begin{tabular}{lcc}
\hline Type & Fuzzy variable & Number of MFs \\
\hline Input (1) & Phase & 9 \\
Input (2) & Frequency & 9 \\
Output & Tuning frequency & 11 \\
\hline
\end{tabular}

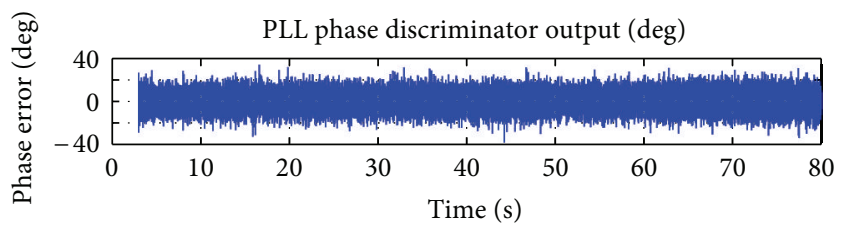

(a)

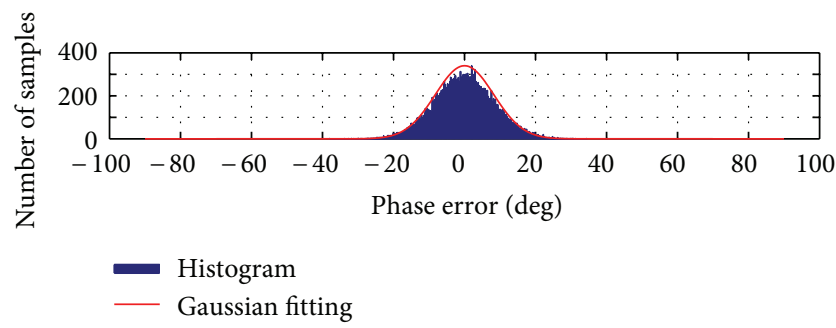

(b)

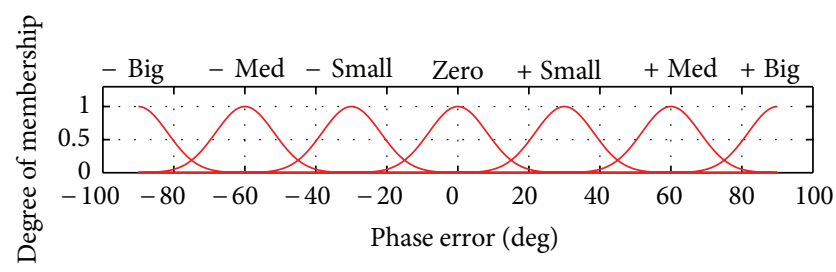

(c)

FIGURE 5: (a) Time domain representation of a PLL phase discriminator output. (b) Histogram and Gaussian approximation. (c) An example of mapping between PDF and MF.

made, and in this case the probability of occurrence, which is described by the PDF, will be replaced by a degree of occurrence sensed by a number of overlapping Gaussian MFs as shown in Figure 5(c). Using the above approach, both phase and frequency error inputs in addition to the NCO tuning frequency output domains are clustered into several overlapping Gaussian fuzzy sets, each of them describes a certain linguistic definition of input or output value (e.g., big, medium, small, zero, etc.). The number of MFs adopted for the fuzzy controller is reported in Table 1. It has been fixed based on multiple simulations of GPS signals conducted with the Spirent GSS7700 hardware simulator [23].

The number of fuzzy sets associated with each fuzzy variable is a design parameter selected according to the required tracking accuracy. How much these contiguous sets should overlap is also a design issue depending on the problem at hand. Too much overlap blurs the distinction between the fuzzy set values, whereas too little can produce excessive overshoot and undershoot. The fuzzy rules that relate all linguistic variables can be expressed as

$$
R_{i} \text { : if } \theta=A_{1}^{i} \& \omega=A_{2}^{i} \text { then } \Delta f=B^{i} \text { for } i \in\{1, L, 81\}
$$

where $\theta, \omega$, and $\Delta f$ are linguistic variables representing the input phase, the frequency, and the output NCO tuning frequency. $A_{1}^{i}, A_{2}^{i}$, and $B^{i}$ are linguistic labels (or fuzzy sets) characterized by a MF and used in the rule number " $i$ " or $R_{i}$. A defuzzification process is utilized to determine a crisp value according to the fuzzy output from the inference mechanism. The centre-average method [7] or Fuzzy centroid, which calculates the centre of the area of the inference mechanism output possibility distribution, is used as Defuzzification strategy in FFPLL. The output, $y$, is obtained as follows:

$$
\bar{y}=\frac{\sum_{i=1}^{n} y_{i} u\left(y_{i}\right)}{\sum_{i=1}^{n} u\left(y_{i}\right)}
$$

where $n$ is the number of fuzzy output sets, $y_{i}$ is the numerical value of the $i$ th output membership function, and $u\left(y_{i}\right)$ represents its membership value at the $i$ th quantization level. The fuzzy rule table providing the human knowledge base of the controller is shown in Table 2.

Gaussian MFs ended by trapezoidal rules were chosen as shown in Figure 6. The variance of each Gaussian function changes according to the signal noise level with $C / N_{0}$ and $T$ used as inputs in (1). The "on the fly" adaptation is further detailed in [24].

The FAMs are designed to act like an FLL for fast frequency tracking recovery in case of a large frequency error indicated by the frequency discriminator. That can be seen in Table 2 in all the rules except when the frequency error is in the zero region. In this case the phase error is first sought, which is quantified by the phase discriminator for accurate phase tracking and to extract the required data message.

The position and width of the output MFs specify the amount of the required NCO tuning frequency for frequency and phase tracking. The ease of controlling and changing fuzzy MFs enables the designer to control the output to satisfy his own experience and requirements. In this case, fuzzy MFs with their connecting rules are selected to provide a small equivalent bandwidth in the case of small errors and wider equivalent bandwidth, should more significant phase and frequency errors occur. The technique used for designing and tuning the Fuzzy rules and MFs and to calculate the equivalent bandwidth is explicitly described in [20].

Using Fuzzy Logic instead of a standard FLL-aided PLL is performed at the cost of an increased computational load, as 81 rules have to be processed in parallel. Therefore it is desirable to eliminate some rules in the FFPLL design for improving computational efficiency. Using INS aiding, the frequency discriminator and its fuzzy rules can be dropped. The modified FFPLL design using INS aiding is next presented. 
TABLE 2: Fuzzy rules.

\begin{tabular}{|c|c|c|c|c|c|c|c|c|c|}
\hline \multirow{2}{*}{$\omega$} & \multicolumn{9}{|c|}{$\theta$} \\
\hline & $-B$ & $-\mathrm{MB}$ & $-\mathrm{M}$ & $-S$ & $\mathrm{Ze}$ & $+\mathrm{S}$ & $+\mathrm{M}$ & $+\mathrm{MB}$ & $+\mathrm{B}$ \\
\hline$-B$ & $+\mathrm{B}$ & $+\mathrm{B}$ & $+\mathrm{B}$ & $+B$ & $+\mathrm{B}$ & $+B$ & $+B$ & $+B$ & $+\mathrm{B}$ \\
\hline$-\mathrm{MB}$ & $+\mathrm{MB}$ & $+\mathrm{MB}$ & $+\mathrm{MB}$ & $+\mathrm{MB}$ & $+\mathrm{MB}$ & $+\mathrm{MB}$ & $+\mathrm{MB}$ & $+\mathrm{MB}$ & $+\mathrm{MB}$ \\
\hline$-\mathrm{M}$ & $+\mathrm{M}$ & $+\mathrm{M}$ & $+\mathrm{M}$ & $+\mathrm{M}$ & $+\mathrm{M}$ & $+\mathrm{M}$ & $+\mathrm{M}$ & $+\mathrm{M}$ & $+\mathrm{M}$ \\
\hline$-S$ & $+\mathrm{SM}$ & $+\mathrm{SM}$ & $+\mathrm{SM}$ & $+\mathrm{SM}$ & $+\mathrm{SM}$ & $+\mathrm{SM}$ & $+\mathrm{SM}$ & $+\mathrm{SM}$ & $+\mathrm{SM}$ \\
\hline $\mathrm{Ze}$ & $-\mathrm{MB}$ & $-\mathrm{M}$ & $-S M$ & $-S$ & $\mathrm{Ze}$ & $+\mathrm{S}$ & $+\mathrm{SM}$ & $+\mathrm{M}$ & $+\mathrm{MB}$ \\
\hline$+\mathrm{S}$ & $-\mathrm{SM}$ & $-\mathrm{SM}$ & $-\mathrm{SM}$ & $-\mathrm{SM}$ & $-\mathrm{SM}$ & $-\mathrm{SM}$ & $-\mathrm{SM}$ & $-\mathrm{SM}$ & $-\mathrm{SM}$ \\
\hline$+\mathrm{M}$ & $-\mathrm{M}$ & $-\mathrm{M}$ & $-\mathrm{M}$ & $-\mathrm{M}$ & $-\mathrm{M}$ & $-\mathrm{M}$ & $-\mathrm{M}$ & $-\mathrm{M}$ & $-\mathrm{M}$ \\
\hline$+\mathrm{MB}$ & $-\mathrm{MB}$ & $-\mathrm{MB}$ & $-\mathrm{MB}$ & $-\mathrm{MB}$ & $-\mathrm{MB}$ & $-\mathrm{MB}$ & $-\mathrm{MB}$ & $-\mathrm{MB}$ & $-\mathrm{MB}$ \\
\hline$+\mathrm{B}$ & $-B$ & $-B$ & $-B$ & $-B$ & $-B$ & $-B$ & $-B$ & $-B$ & $-B$ \\
\hline
\end{tabular}

\pm B: \pm big; \pm MB: \pm medium big; \pm M: \pm medium; \pm SM: \pm small medium; \pm S: \pm small; Ze: zero.

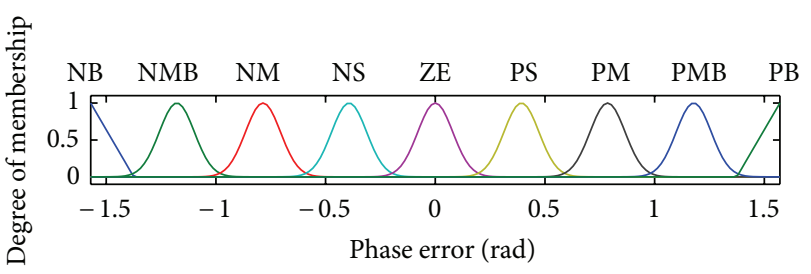

(a)

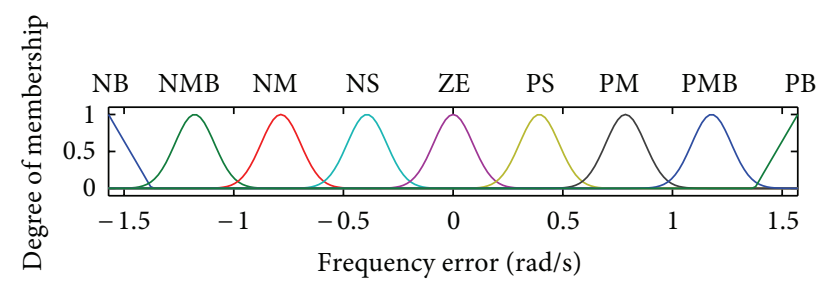

(b)

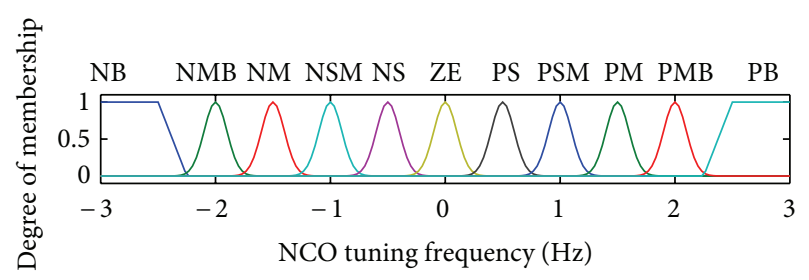

(c)

FIGURE 6: Inputs and output membership functions.

\section{INS-Assisted FFPLL Design}

Coupling GPS and INS signals provides a good solution for improving guidance of high-rate maneuvering platforms. The concept links GPS and INS to allow aiding. Aiding can be performed in several ways. GPS can be used to periodically update the state vector of the navigation filter for calibrating the errors inherent to inertial sensors. The INS can improve tracking and reacquisition time by providing a reference signal to the GPS receiver tracking loops. GPS and inertial signals can be coupled using KF techniques to fuse navigation information and handle short GPS outages, signal interferences, or intentional jamming [25]. The levels of performance achieved depend on the grade of the INS and the GPS satellites visibility and are driven generally by the required accuracy or missile-target miss distance. Coupling becomes necessary on missile systems and projectiles in order to mitigate the effects of extreme dynamics, rapidly changing GPS visibility and potential jamming [26, 27].

Based on different data fusion strategies, GPS/INS integrated systems can be classified into three types: loosely coupled GPS/INS, tightly coupled GPS/INS, and ultratight GPS/INS integration [28]. As the main objective of the proposed research is to aid the GPS tracking loop with INS data, the coupling filter corresponds to an ultra-tight integration architecture [29]. This combines the GPS signal tracking functions, the INS data, and the integration filter into a single estimation algorithm. In this algorithm the INS measurements are fed back to the receiver as seen in Figure 2 for decreasing GPS signal tracking errors and enhancing the final GPS positioning performance.

During GPS outages, the INS-derived Doppler can be continuously estimated and used for assisting GPS tracking loops by directly pulling the receiver back to tracking mode without passing through an acquisition step if signal lock is lost due to interference or jamming. The INS data of interest to assist the tracking loop is the INS Doppler, which can be extracted for the proposed GPS/INS integration scheme through the sequence now explained. Using ephemeris data calculated with the incoming data bits extracted during the signal tracking phase, the satellite positions and velocities are calculated in the Earth-Centered, Earth-Fixed (ECEF) coordinate frame. This information is then combined with the calculated ECEF navigation coordinates using INS only in a strapdown integration scheme to estimate the associated Doppler through the steps described below [30].

Firstly, the line of sight (LOS) between the $k$ th satellite and the INS processed location is calculated as

$$
\begin{gathered}
\mathbf{e}_{k}=\frac{1}{r_{k}}\left(\mathbf{x}_{\mathrm{INS}}-\mathbf{x}_{s_{k}}\right), \\
r_{k}=\sqrt{\left(\mathbf{x}_{\mathrm{INS}}-\mathbf{x}_{s_{k}}\right)^{T}\left(\mathbf{x}_{\mathrm{INS}}-\mathbf{x}_{s_{k}}\right)},
\end{gathered}
$$


where $r_{k}$ is the distance between the $k$ th satellite and the INS coordinates, and $\mathbf{e}_{k}$ is the unit vector of the LOS between the $k$ th satellite and the INS position. $\mathbf{x}_{\text {INS }}$ represents the INS coordinates in the ECEF, while $\mathbf{x}_{s_{k}}$ represents the $k$ th satellite position in ECEF. Secondly, INS Doppler $\left(f_{d \text {-INS }}\right)$ is derived from (4) using

$$
f_{d-\mathrm{INS}, k}=\frac{1}{\lambda_{L 1}} \mathbf{e}_{k}^{T}\left(\mathbf{v}_{\mathrm{INS}}-\mathbf{v}_{s_{k}}\right),
$$

where $\mathbf{v}_{\text {INS }}$ and $\mathbf{v}_{s_{k}}$ are the INS-derived velocity and the $k$ th satellite velocity in the ECEF, respectively. $\lambda_{L 1}$ is the GPS L1 frequency wavelength.

The INS-estimated Doppler is inherently corrected for a small earth rotation through the strapdown mechanization process. However, other small errors such as satellite and receiver clock errors and ionospheric errors are neglected assuming that any errors in frequency aiding to the tracking loop is compensated using the robust process of fuzzy phase tuning.

The use of INS-estimated Doppler reduces the complexity of the fuzzy tracking loop by using only the phase discriminator output as a single input to the fuzzy processor and the INS Doppler for tuning the frequency. The INS Doppler is added to the IF frequency $\left(f_{\mathrm{IF}}\right)$ and is then subtracted from the NCO generated carrier frequency $\left(f_{c \text {-NCO }}\right)$. The resulting frequency corresponds to a frequency correction signal $\left(\Delta f_{\text {correction }}\right)$ that is used to adjust the NCO tuning frequency $(\Delta f)$. Therefore only 9 fuzzy rules, which correspond to the phase tuning cells in Table 2, instead of the 81 rules in the FFPLL original design, are used for the input fuzzy sets. The complete INS-assisted FFPL is shown in Figure 7.

Before assessing the performance of the FFPLL and the INS-aided FFPLL with experimental data collections, simulation tests and comparison with Kalman filter tracking (Section 4), it is of interest to perform a qualitative comparison with other recently published methods. The general tracking problem always involves some form of Bayesian filtering. If the conditional probability density functions are known to be jointly Gaussian and that the observations relate linearly to the tracked state variables, then the appropriate and optimum Bayesian filter is the standard Kalman filter which has been extensively published since the 1960s. If the observations relate to the state variables in a mildly nonlinear fashion then the conditional densities are only approximately Gaussian. A practical implementation of a Bayesian filter is then the family of extended Kalman filters, scented variants, and so forth. If the relation of the observations to the state variables deviates significantly from being linear, then it is necessary to use numerical integration to perform the Bayesian filter iterations. A practical algorithm that has been recently adopted for this purpose is the particle filter [31]. However, the applicability of these methods is based on the assumption that the conditional density functions are known. If the density functions are not explicitly known then a practical alternative is to use Fuzzy Logic-based Bayesian filters. Fuzzy Logic membership functions allow the filter to be designed in a robust albeit suboptimal way that is based on reasonable statistical modeling assumptions
TABLE 3: Error characteristics of Litton LN-200 IMU.

\begin{tabular}{|c|c|c|}
\hline Sources & 1- $\sigma$ error & Units \\
\hline Gyro bias & 0.35 & $\mathrm{deg} / \mathrm{hr}$ \\
\hline Gyro scale factor & 100 & ppm \\
\hline Gyro misalignment & 0.1 & $\operatorname{mrad}$ \\
\hline Gyro g drift & 0.5 & $\operatorname{deg} / \mathrm{hr} / \mathrm{g}$ \\
\hline Gyro g ${ }^{2}$ drift & 0.05 & $\mathrm{deg} / \mathrm{hr} / \mathrm{g}^{2}$ \\
\hline Gyro random walk & 0.05 & $\mathrm{deg} / \sqrt{\mathrm{h} r}$ \\
\hline Accel. bias & 0.07 & $\mathrm{mg}$ \\
\hline Accel. scale factor & 300 & ppm \\
\hline Accel. misalignment & 0.1 & $\operatorname{mrad}$ \\
\hline Accel. random walk & 0.03 & $\mathrm{~m} / \mathrm{s} / \sqrt{ } \mathrm{hr}$ \\
\hline
\end{tabular}

[32]. As demonstrated in this paper, good dynamic tracking performance is achievable with minimal prior modeling information. Statistical modeling is difficult in the context of capricious jamming signals which gives impetus to the use of fuzzy Logic controllers.

\section{Experimental Test Description}

The main test to assess the proposed INS-assisted FFPLL was conducted using real GPS signals, which were processed with real INS data processing and a GPS jammer. However, because it is very hard to conduct an experimental test that includes missile level dynamics and that is still consistent with the scope of the research, simulated GPS and INS signals are first used with high dynamics to assess the performance and the validity of the proposed algorithm for missile applications.

4.1. Simulator Tests. The proposed algorithm was first tested using the Spirent GSS7700 simulator [23]. The simulator is capable of providing simulated inertial sensors measurements with a controllable output rate that are precisely synchronized with the GPS RF signals. According to the type of application adopted in this work, the tactical grade Litton LN-200 inertial measuring unit (IMU) has been simulated and used. The error characteristics of this IMU are shown in Table 3.

Although it is proposed to perform a complete GPS/INS ultra-tight integration, RF signal processing is developed up to the level of signal tracking and solved the navigation data bits to obtain the GPS time for synchronization purposes. Simulated IMU measurements are processed to calculate the INS solution through the strapdown mechanization process. To get close to the ultra-tight integration objective, the IMU and GPS simulated data are tightly integrated through an extended Kalman filter (EKF) [30] to provide a corrected estimate of the INS solution. The corresponding INS Doppler is estimated as per (4) and (5) using the GPS data provided by the simulator. As a final step, the calculated INS-derived Doppler is used separately to aid the RF signal tracking process through FFPLL. This approach is clearly illustrated in Figure 8 as a modified version of the ultra-tight integration scheme introduced in Section 1. 


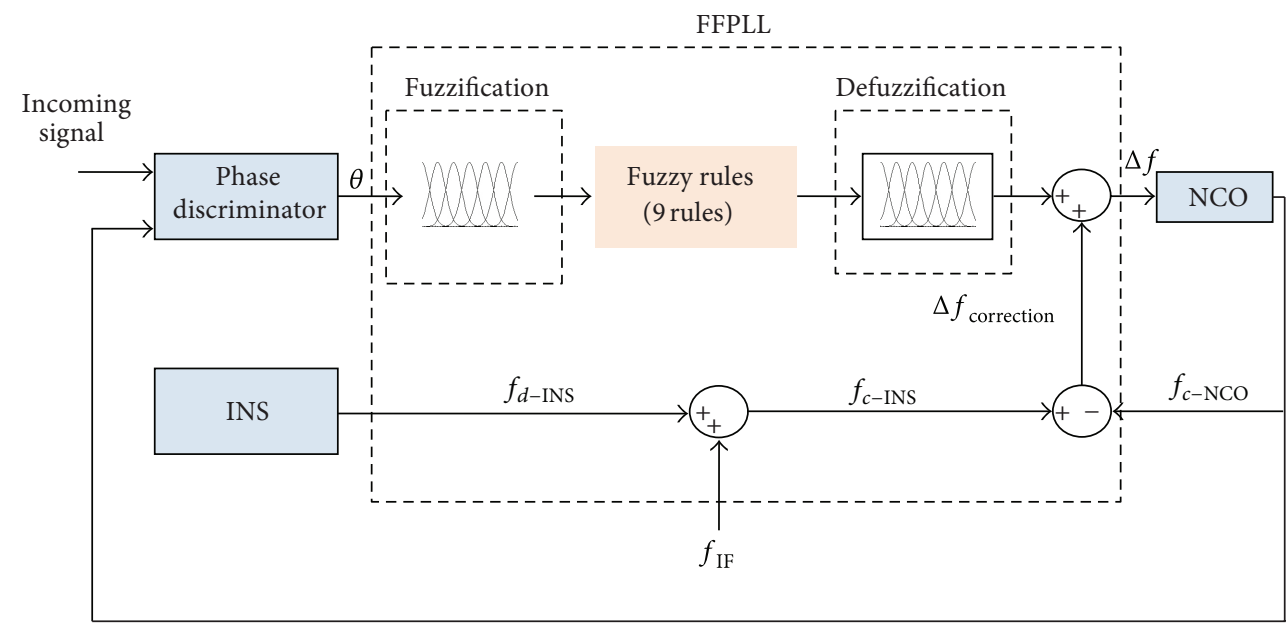

FIGURE 7: INS-assisted FFPLL.

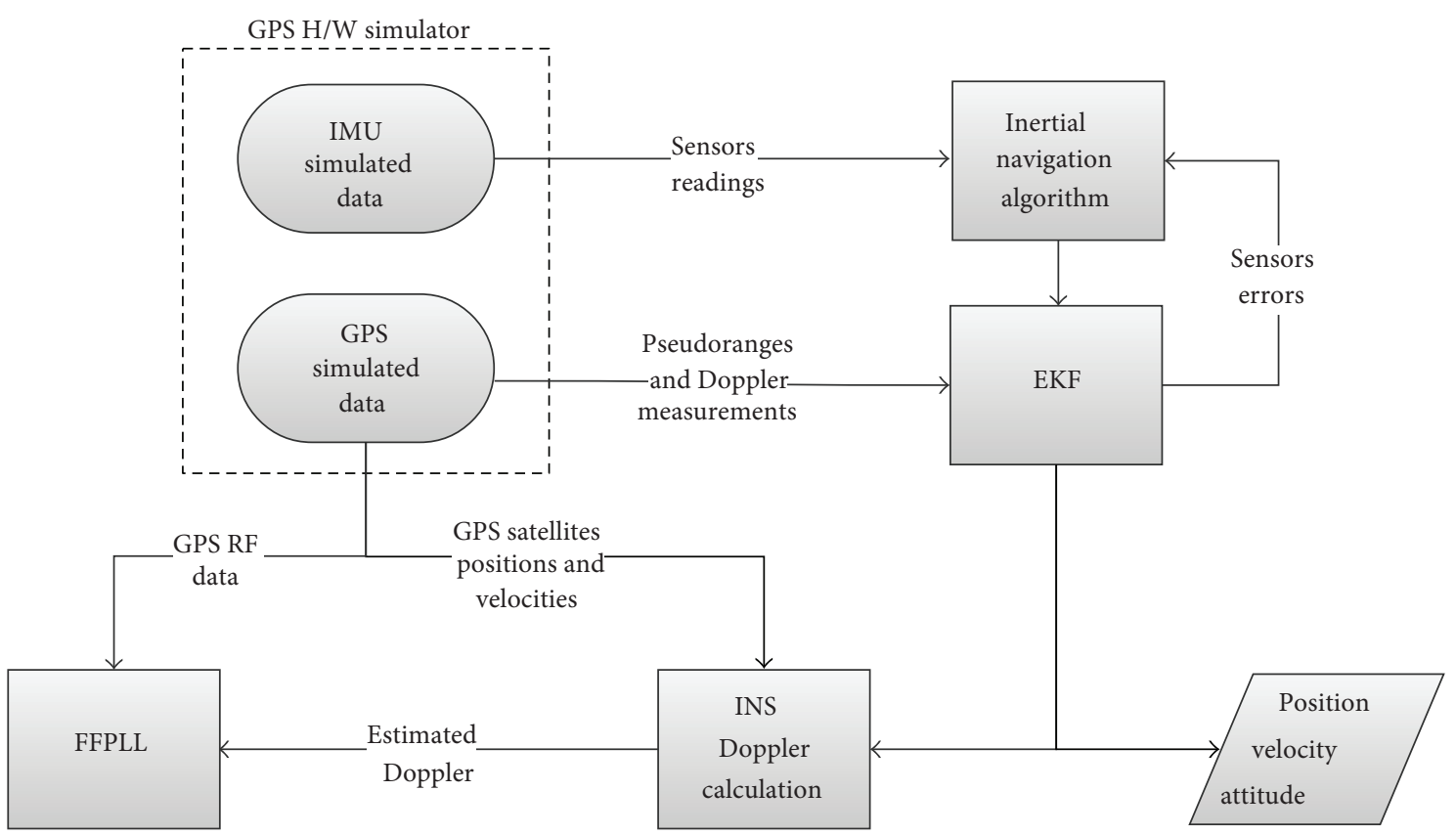

FIgURE 8: Process used to calculate INS Doppler to aid FFPLL.

The test procedure and process algorithm (see Figure 8) are conducted through a large number of scenarios that show consistent results. One of these scenarios is presented herein.

The scenario considered here comprises the effect of missile manoeuvres near an interference source. During the manoeuvre, the GPS signal $C / N_{0}$ changes with the distance from the interference source. The missile velocity in this scenario is increased to reach $300 \mathrm{~m} / \mathrm{s}$, performing hard manoeuvres with acceleration up to $8 \mathrm{~g}$ and jerks up to $50 \mathrm{~g} / \mathrm{s}$. The dynamic profile of this scenario is illustrated in Figure 9, while Figure 10 shows the 3D plot of the missile's trajectory and its manoeuvres near the jammer.

A CW jammer producing $45 \mathrm{~dB}$ (J/S) of jamming signal is simulated in this scenario. Estimated $C / N_{0}$ changes as the missile performs an evasive manoeuvre to escape this jamming are shown in Figure 11. As shown in [20], the
FFPLL is capable of maintaining signal lock regardless of the missile dynamics or if low or moderate interference levels are applied. Very accurate Doppler estimates, extracted from tactical grade IMU measurements, are helping to provide continuous signal tracking even if a high level of interference is applied. When tracking is switched from FFPLL to INS-assisted FFPLL, course frequency tracking is totally dependent on INS-provided Doppler, and hence the fuzzy processor is responsible only for tracking frequency errors by fine tuning and for providing phase tracking. Using a one input fuzzy processor reduces drastically its complexity and the number of fuzzy rules processed in parallel. Consequently the computational load gets reduced as will be described later.

Figures 12 and 13 show the estimated Doppler for PRN 24 for the duration of the entire scenario and a magnified version for better illustration, respectively. The Doppler is 


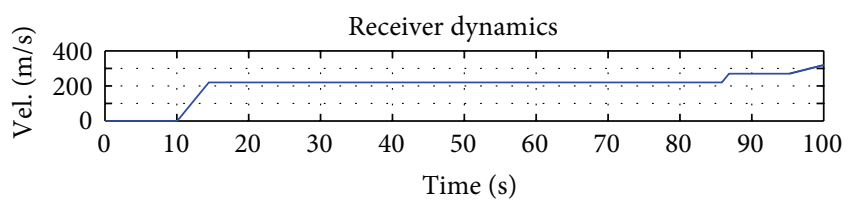

(a)

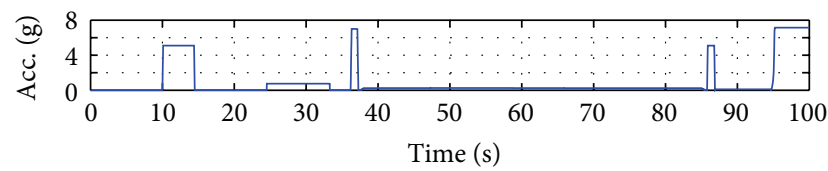

(b)

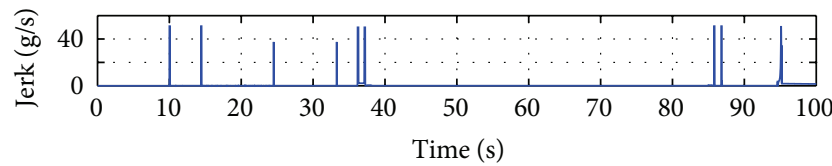

(c)

FIGURE 9: Receiver dynamics profile.

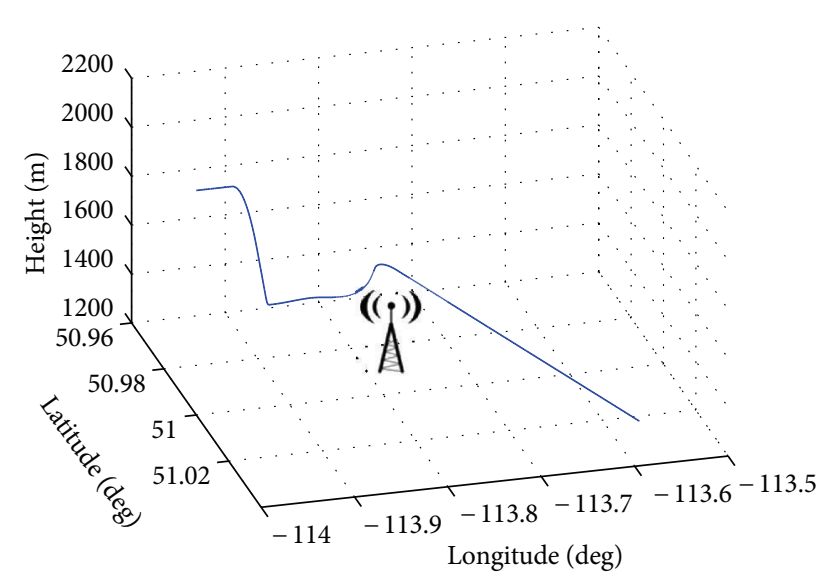

FIGURE 10: 3D plot of the missile manoeuvres near an interference source.

estimated during signal tracking using KF + INS, FFPLL, and FFPLL + INS. Figure 14 shows the estimated Doppler standard deviation using these three algorithms. At epoch 30, GPS time is extracted under proper signal tracking using the original FFPLL, and synchronization is performed with INS aiding data to switch from the original FFPLL to FFPLL + INS. The Kalman filter used here is based on the model described in [26-28] and uses discriminator outputs as measurement updates. It is also modified to accept INS Doppler aiding for a fair comparison with the INS-assisted FFPLL. The INS-assisted Kalman filter is labeled KF + INS.

From the previous results, it can be seen that the INSassisted FFPLL algorithm has successfully passed the validity test by providing continuous tracking during combined incidence of very high dynamics and variable jamming levels. As shown in Figure 13 for PRN 24, thanks to accurate Doppler estimates from the simulated Litton IMU, the estimated Doppler is more accurate than the one estimated with the standalone FFPLL. A more complete assessment of the

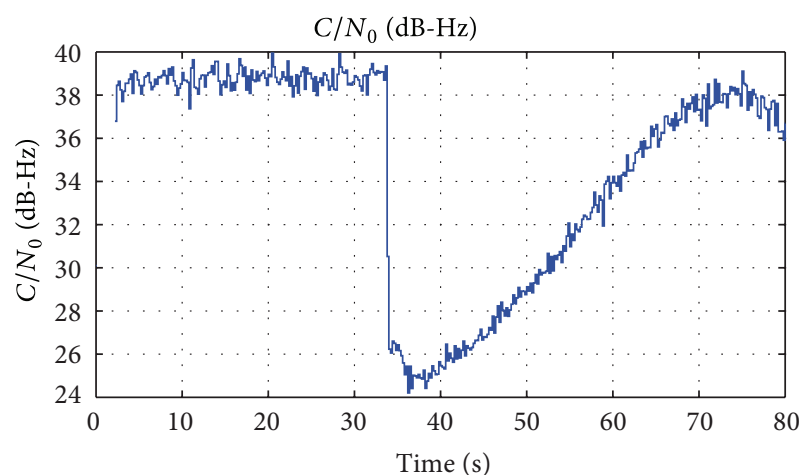

Figure 11: $C / N_{0}$ estimated for PRN 24 during missile manoeuvres.

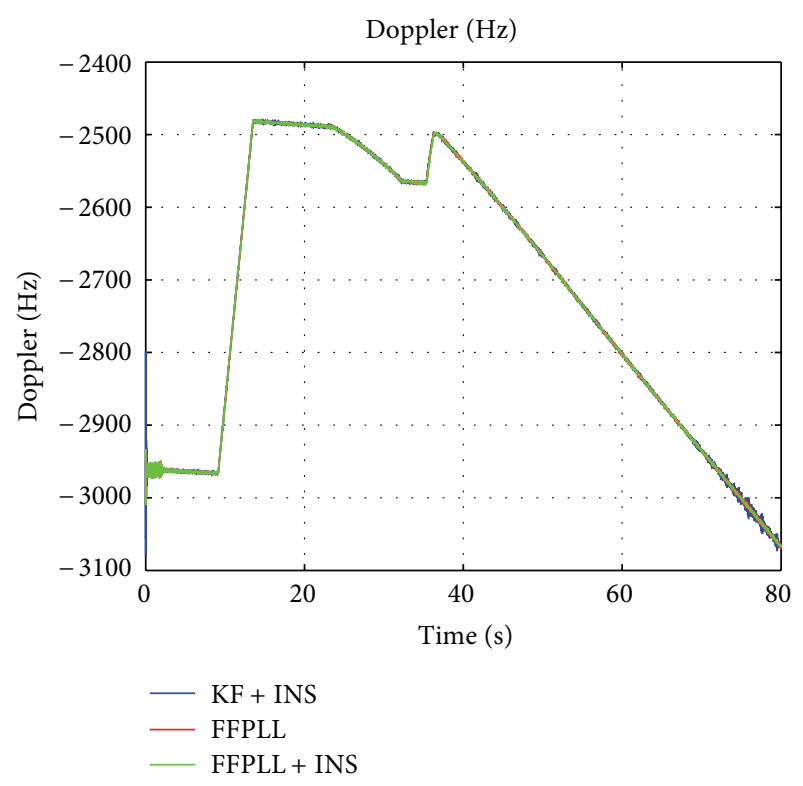

FIGURE 12: Estimated Doppler for PRN 24 using KF + INS, FFPLL, and INS + FFPLL.

proposed algorithm is given in the next section where real GPS and IMU signals have been collected and analyzed.

4.2. Experimental Test Description. In order to test the proposed signal tracking architecture using real GPS and INS data, experimental tests have been conducted in a vehicle. Photos of the testing equipment are shown in Figures 15 and 16. They comprise a GPS antenna and the NovAtel SPAN Honeywell HG1700 system. The lever arm was measured during the experiment. To remain as close as possible to the scope of the research, dynamics conditions limited by the vehicle capability have been reproduced during the data collection. Logging and control systems were loaded in the vehicle. The complete test setup is illustrated in Figure 17.

Rover data was collected with a single antenna using two separate setups. The NovAtel 702 GG antenna was used to collect GPS signals, and its output was split between an OEM4 dual frequency GPS receiver, which was connected to a tactical grade IMU (Honeywell HG1700 [33]), and a National 


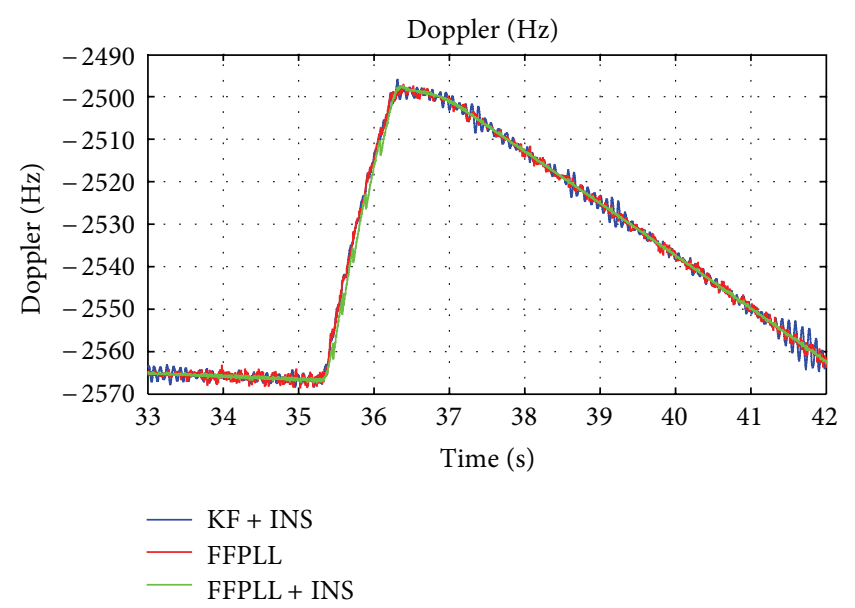

FIGURE 13: Estimated Doppler for PRN 24 using KF + INS, FFPLL, and INS + FFPLL (magnified).

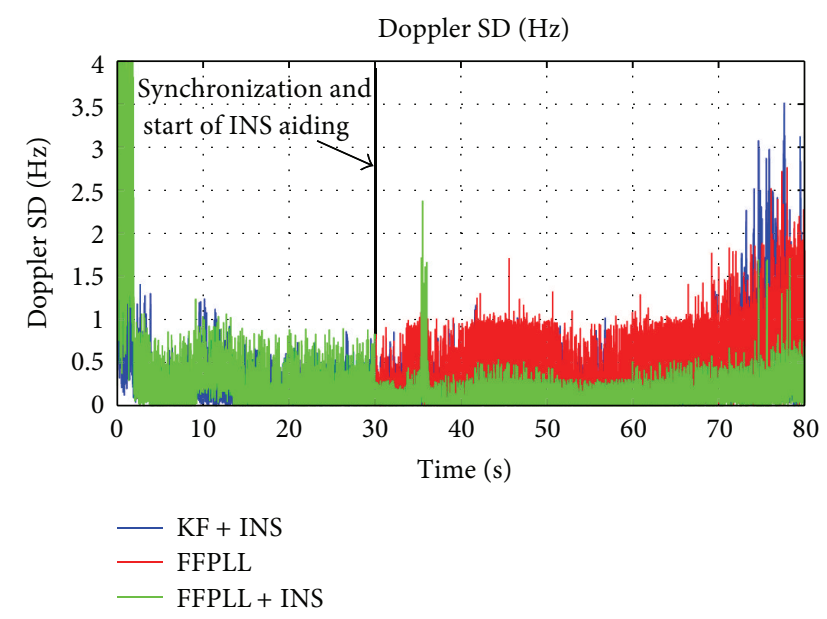

Figure 14: Estimated Doppler SD for PRN 24 using KF + INS, FFPLL, and INS + FFPLL.

TABLE 4: Equipment used for field data collection.

\begin{tabular}{lc}
\hline Number & Equipment \\
\hline 1 & NovAtel GPS receiver (2) \\
2 & Honeywell HG1700 IMU \\
3 & National Instruments RF front end \\
4 & NovAtel GPS antenna (2) \\
5 & Handheld commercial GPS jammer \\
\hline
\end{tabular}

Instruments RF front end. The front end was controlled by a PC used for RF sample data logging. The post-processed GPS/INS solution was used as reference for assessing the performance of the INS-assisted FFPLL. GPS signals were jammed using a handheld short range GPS jammer through cabling to the National Instruments RF front end as shown in Figure 18. The cable connection was used to avoid over the air jamming. Table 4 lists the equipment used.

4.3. GPS/INS Reference Solution. The Honeywell HG1700 AG58 has a 1-degree per hour gyro bias stability. The other

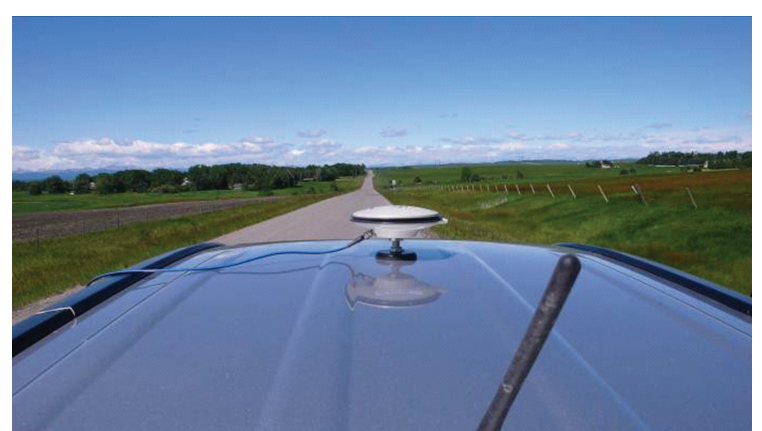

FIGURE 15: NovAtel 702 GG antenna mounted on the vehicle rooftop.

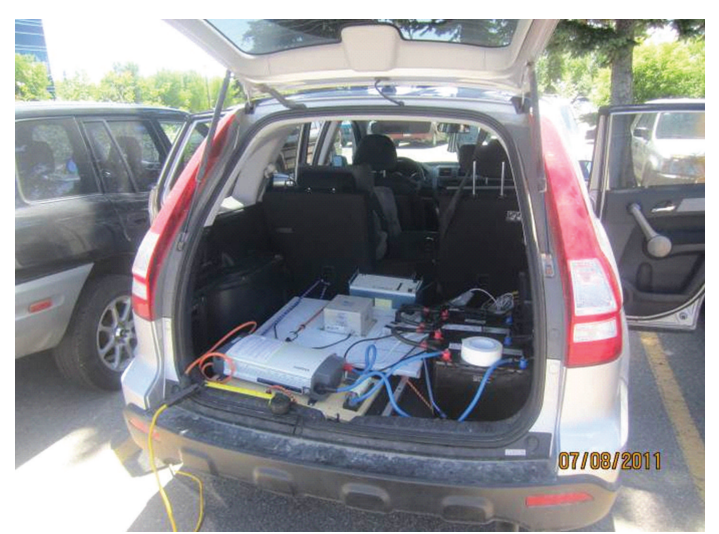

FIGURE 16: Tactical grade IMU (Honeywell HG1700) shown inside the vehicle.

system specifications are given in Table 5. INS Doppler values were estimated using postprocessed vehicle positions and velocities computed with the postprocessing software Inertial Explorer [34]. Tight coupling of GPS dual frequency signals from a stationary GPS base station and the vehicle-mounted receiver with inertial data was performed after solving for initial static GPS/IMU alignment within the software. Furthermore proper stochastic modelling of the HG1700 was set prior to processing. In the tightly coupled approach, only one Kalman filter was used, which enabled the filter to be updated using as little as one satellite measurement. Along with the navigation solution, accelerometers bias and gyroscope drifts were continuously estimated. The lever arm between the IMU and the GPS rover antenna was surveyed to within a centimetre level using a Leica reflectorless total station [35] as shown in Figure 19. The maximum baseline length was about $25 \mathrm{~km}$.

Two approaches have been used for the data postprocessing. The first approach is the use of differential GPS data with forward and backward smoothing using a RauchTung-Striebel smoother [36] to minimize GPD outage errors and to obtain the best possible reference INS solution. The Inertial Explorer software internal filter accuracy estimation gave mean standard deviations of about $0.01 \mathrm{~m}$ for the position solutions and $0.004 \mathrm{~m} / \mathrm{s}$ for the speed.

To test a more realistic scenario where it is very hard to use differential GPS or smoothing for real time long range missile 


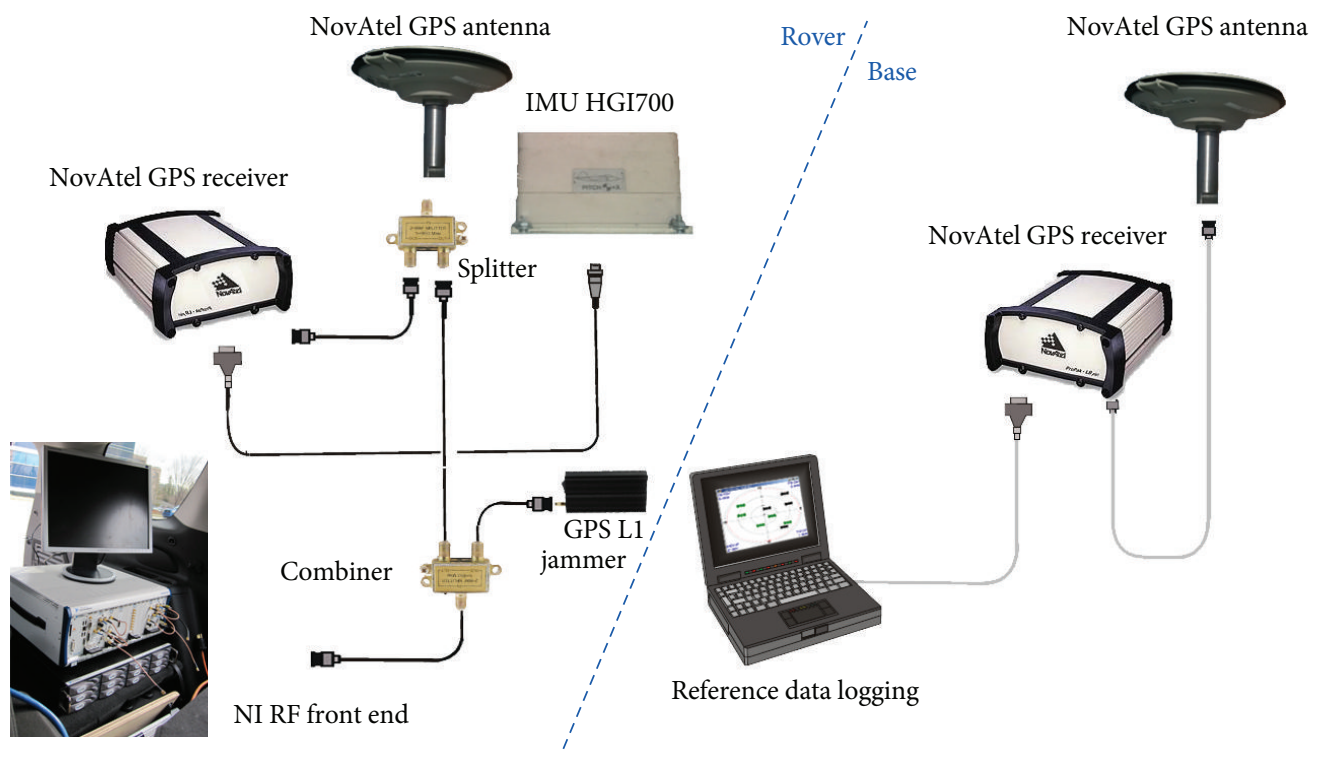

Figure 17: Test setup.

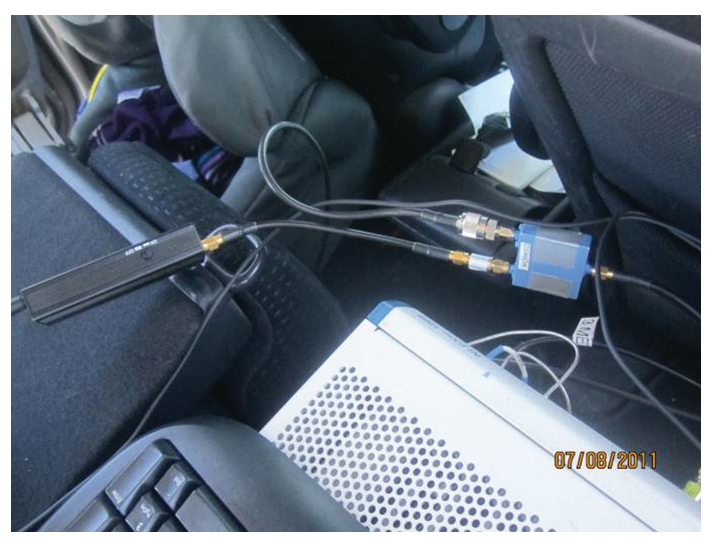

FIGURE 18: Handheld jammer signal combined with GPS signal via cabling and connected to the logging system.

TABLE 5: HG1700 IMU system specifications.

\begin{tabular}{lcc}
\hline Description & & Units \\
\hline Output data rate & $100 / 600$ & $\mathrm{~Hz}$ \\
Gyro input range & $\pm 1,000$ & $\mathrm{deg} / \mathrm{sec}$ \\
Gyro rate scale factor & 150 & $\mathrm{ppm}$ \\
Gyro bias (one sigma) & 1 & $\mathrm{deg} / \mathrm{hr}$ \\
Gyro angular random walk & 0.125 & $\mathrm{deg} / \sqrt{\mathrm{hr}}$ \\
Accel. range & \pm 50 & $\mathrm{~g}$ \\
Accel. linearity & 500 & $\mathrm{ppm}$ \\
Accel. scale factor & 300 & $\mathrm{ppm}$ \\
Accel. bias & 1.0 & $\mathrm{mg}$ \\
\hline
\end{tabular}

applications, the INS solution was recalculated using a second approach where only the vehicle-mounted GPS data was used in a forward solution with a Precise Point Positioning (PPP) method [37]. The Inertial Explorer software internal filter for this approach gave a mean standard deviation of

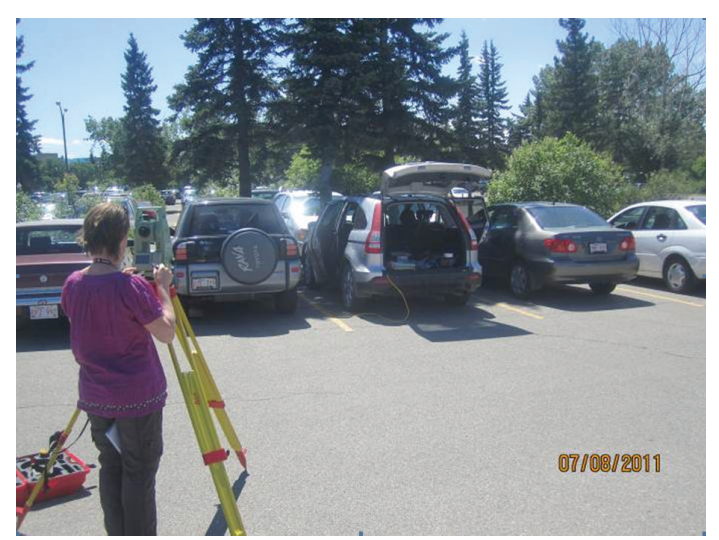

FIGURE 19: Lever arm surveying using Leica TCR705 reflectorless total station.

$0.17 \mathrm{~m}$, a maximum of $1.16 \mathrm{~m}$ for the position solutions, and $0.01 \mathrm{~m} / \mathrm{s}$ with a maximum of $0.09 \mathrm{~m} / \mathrm{s}$ for the speed. These two approaches are described in Figure 20 in term of the differences in INS solution estimates for the positions, while the position and velocity results are shown in Figure 21.

As a following step, the INS Doppler was calculated as described earlier and used as an aiding signal for the FFPLL, which is processed separately. An example of INS Doppler differences between the two approaches for PRN 16 is shown in Figure 22. The experiment is also described in the stepwise flow diagram shown in Figure 23.

4.4. INS Doppler. In order to insure that only INS-derived Doppler were extracted from the tightly coupled solution during jamming periods, artificial outages were simulated in post-processing during the periods of jamming. Indeed the wired GPS jammer perturbed only the RF samples collected by the NI front end and not the GPS receiver used for the INS 


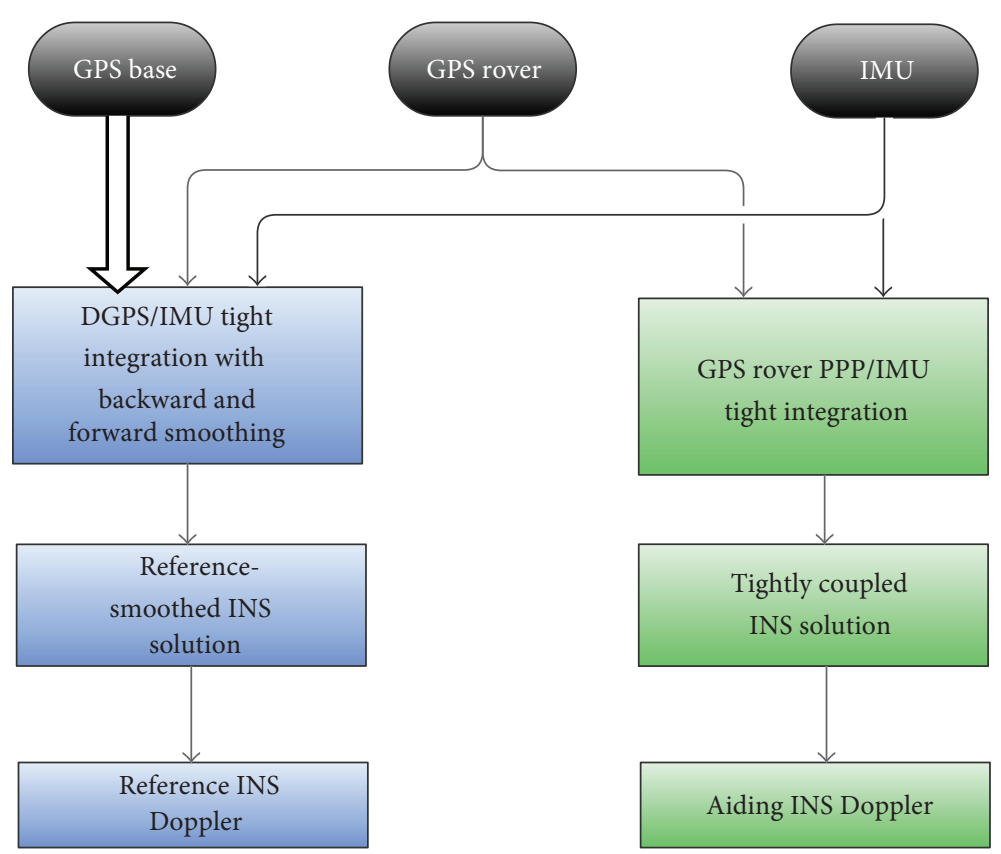

FIgURE 20: Two approaches used to calculate the INS reference and aiding Doppler values.

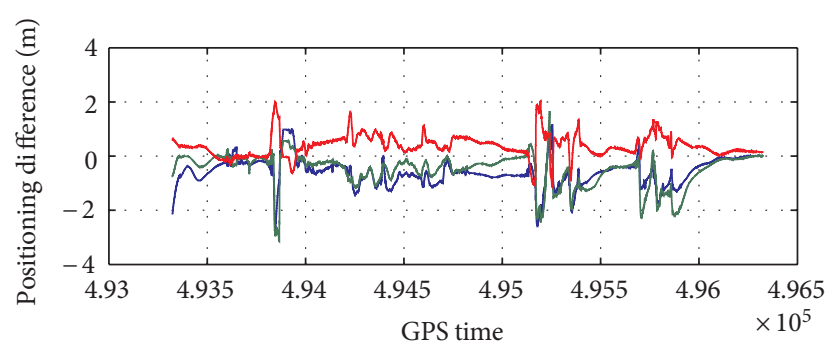

$-\operatorname{ECEF} X$
$-\operatorname{ECEF} Y$
$\operatorname{ECEF} Z$

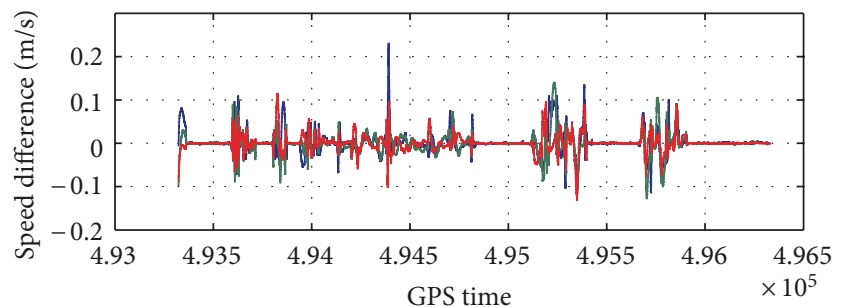

ECEF $V x$
ECEF $V y$
ECEF $V z$

(a)

(b)

FIGURE 21: Differences between INS velocity and position solutions obtained with the GNSS differential approach and the Precise Point Positioning approach.

equipment. This can be seen in Figure 17, where the jammer RF signal is combined with GPS signals only after the splitter used for feeding the NovAtel receiver.

4.5. RF Data. The RF data was down converted and saved for post-processing. The GPS signals were collected using complex sampling of frequency $f_{s}=5 \mathrm{MHz}$ at intermediate frequency $f_{\mathrm{IF}}=0.42 \mathrm{MHz}$.

The "over-the-air" jammer, which is part of the test setup shown in Figure 17, produces a CW signal nearly centered at L1 but with a $50 \mathrm{~dB}$ higher power than the nominal GPS power. As it is explicitly explained in [20], this power is high enough to saturate the receiver's amplifier and distort the signal during jamming periods. To increase the safety factor and to avoid any issues with its wireless use, the jammer was used with a proper cable connection and all induced jamming signal passed through the connecting cables as shown in Figure 18.

\section{Data Processing and Results}

GPS RF samples were post-processed and tracked using different algorithms, as described in Table 6. The bandwidths used for classical tracking schemes correspond to the maximum bandwidths for stable tracking, $18(\mathrm{~Hz})$ in this case [4], for increasing the chance of signal reacquisition after the jamming period and to provide a fair comparison with the proposed INS-aided FFPPL.

The Kalman filter used here is based on the model presented in [38, 39] and uses discriminators outputs as a measurements update. It was also modified to accept INS 
TABLE 6: Algorithms used for comparative analysis of GPS tracking loops.

\begin{tabular}{|c|c|c|c|c|}
\hline & Standard tracking loop & Kalman filter based & Fuzzy Logic & INS assisted \\
\hline (1) PLL (18 Hz) & $x$ & & & \\
\hline (2) FLL $(18 \mathrm{~Hz})$ assisted PLL $(18 \mathrm{~Hz})$ & $x$ & & & \\
\hline (3) INS/KF & & $x$ & & $x$ \\
\hline (4) INS/PLL (18 Hz) & $x$ & & & $\times$ \\
\hline (5) INS/FLL (18 Hz) assisted PLL (18 Hz) & $x$ & & & $x$ \\
\hline (6) FFPLL & & & $x$ & \\
\hline (7) INS/FFPLL & & & $x$ & $x$ \\
\hline
\end{tabular}

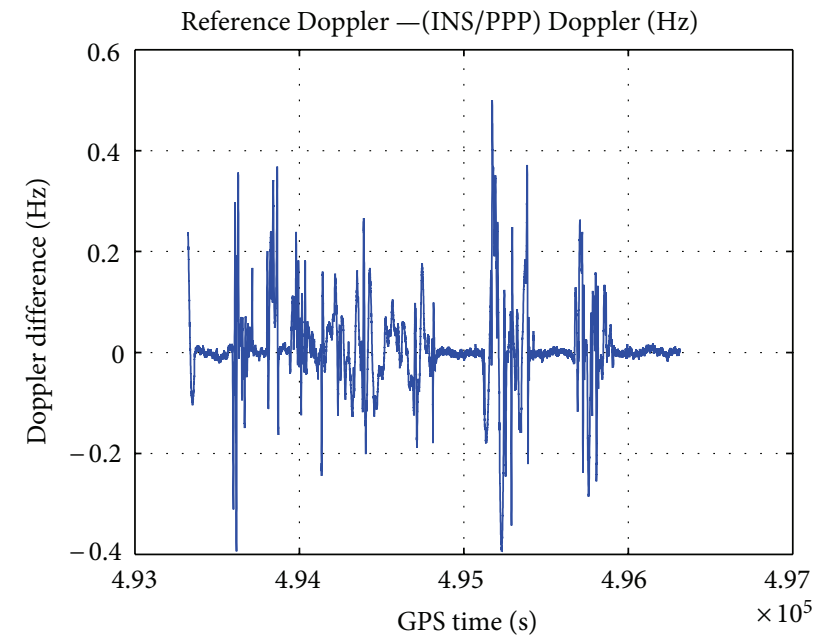

FIGURE 22: Differences between INS Doppler values obtained with the GNSS differential approach and the Precise Point Positioning approach for PRN 16.

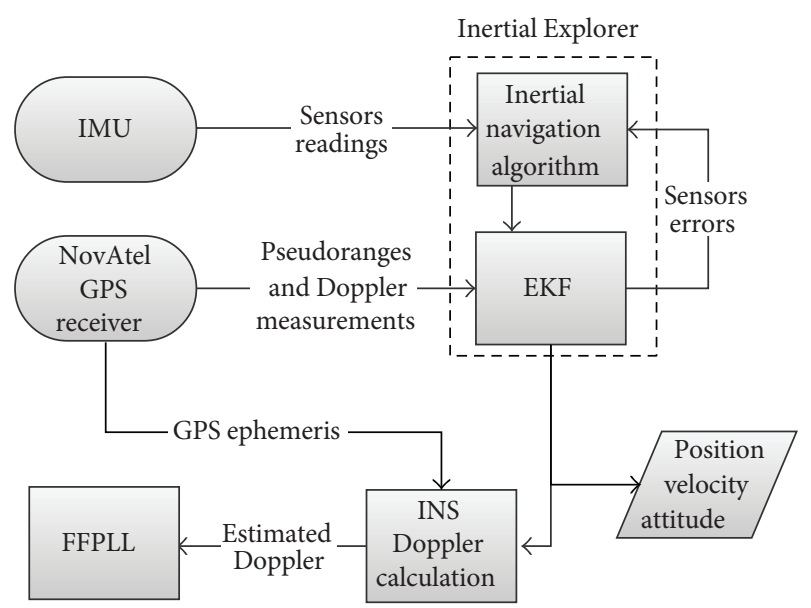

FIGURE 23: Step wise flow diagram and aiding Doppler calculation process.

Doppler aiding for a fair comparison with the INS-assisted FFPLL.

The chosen criteria for assessing the proposed tracking scheme are summarized as follows:

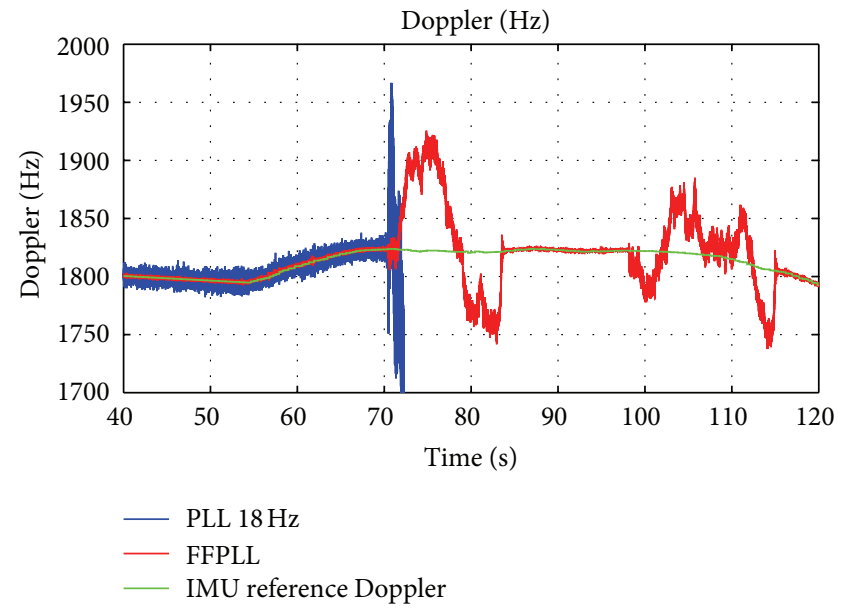

Figure 24: Estimated Doppler for PRN 13 using standard PLL $18 \mathrm{~Hz}$ and FFPLL.

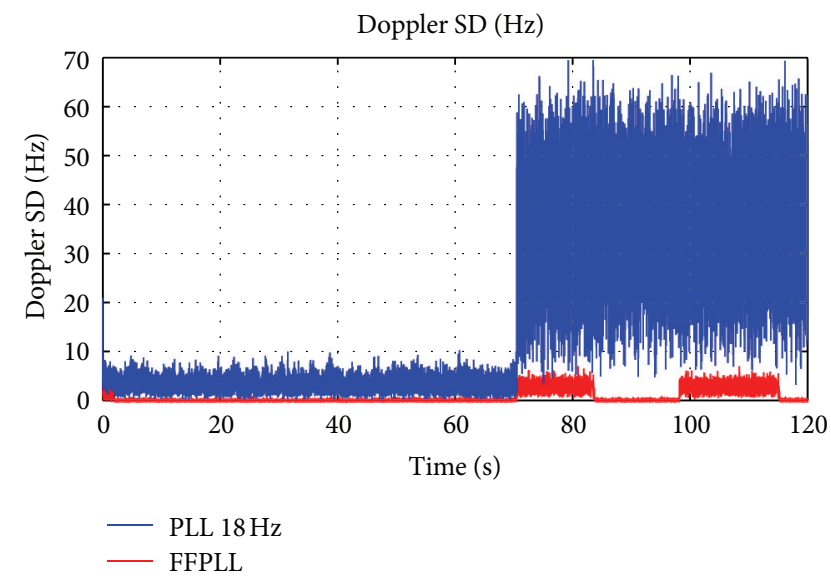

Figure 25: Estimated Doppler SD for PRN 13 using PLL $18 \mathrm{~Hz}$ and FFPLL.

(i) tracking robustness under different dynamic conditions;

(ii) tracking quality by measuring noise distribution modulated on the estimated carrier Doppler;

(iii) ability to mitigate signal interference;

(iv) signal reacquisition ability and time;

(v) Computational load. 


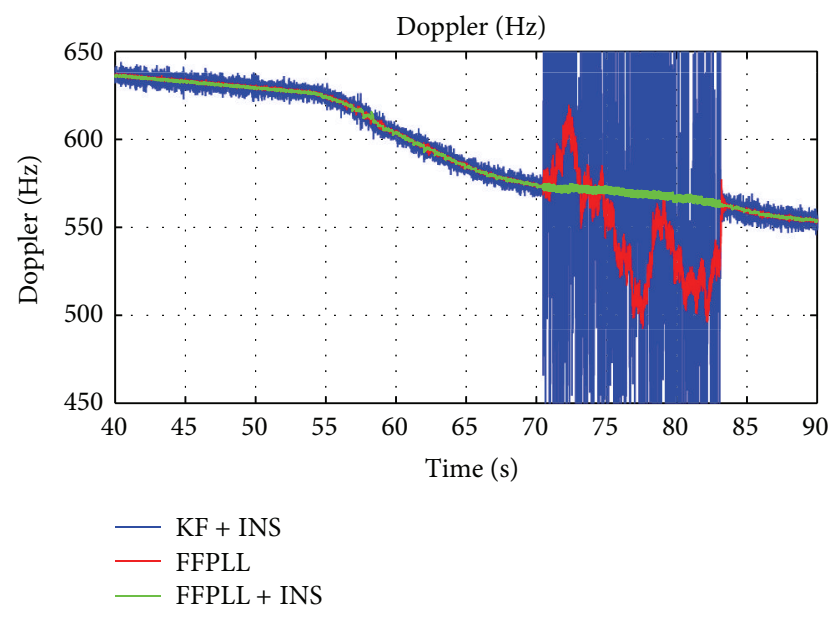

Figure 26: Estimated Doppler for PRN 16 using KF + INS, FFPLL, and INS + FFPLL.

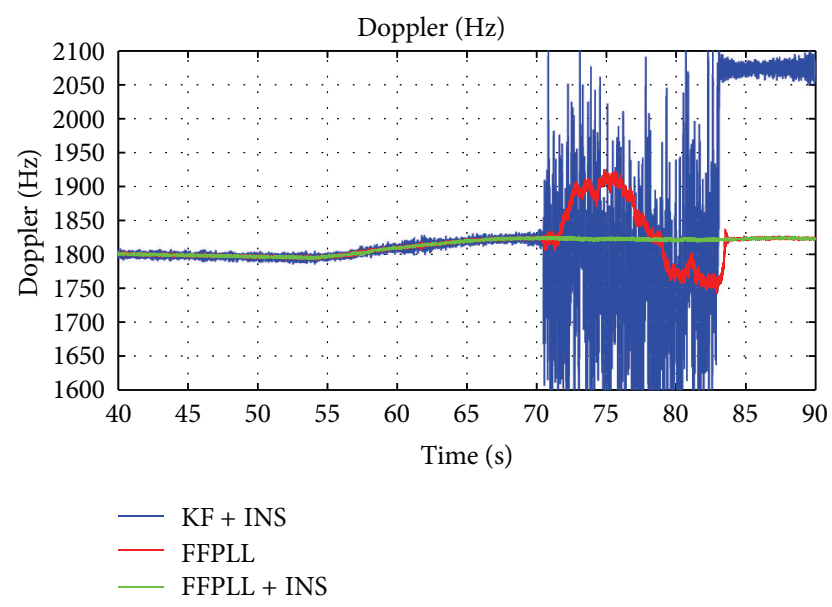

FIgURe 27: Estimated Doppler for PRN 13 using KF + INS, FFPLL, and FPLL + INS.

All the above points of assessment, except the computational load, can be investigated by analyzing the estimated Doppler during signal tracking, taking into consideration that, when the system is in tracking mode, it tracks the signal phase as well. During processing, this estimated Doppler is first compared to the INS reference Doppler, calculated using (5), to ensure that the solution is correct and not biased.

An analysis of the tracking results for two specific GPS signals, PRN code 16 and 13, which were tracked at elevations of about 30 degrees and 60 degrees, respectively, using standard and nonstandard tracking algorithms, is conducted. The associated results are assessed in a concise manner, as all tracking results for the other available GPS signals show consistent results and lead to the same conclusion.

After processing RF data using the approaches listed in Table 6, it was found that none of them were able to track the GPS signal phase during jamming periods. Moreover it was observed that the standard tracking schemes, even with INS aiding, were not able to reacquire the signal and

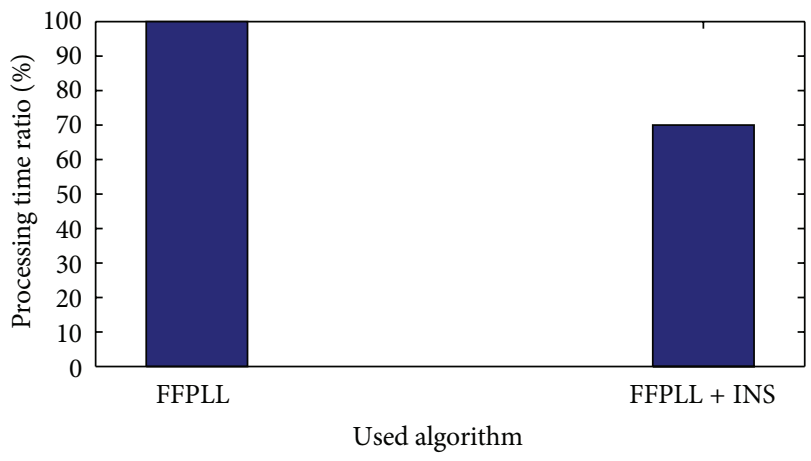

FIGURE 28: Average fuzzy processing time ratio for standalone and assisted FFPLL.

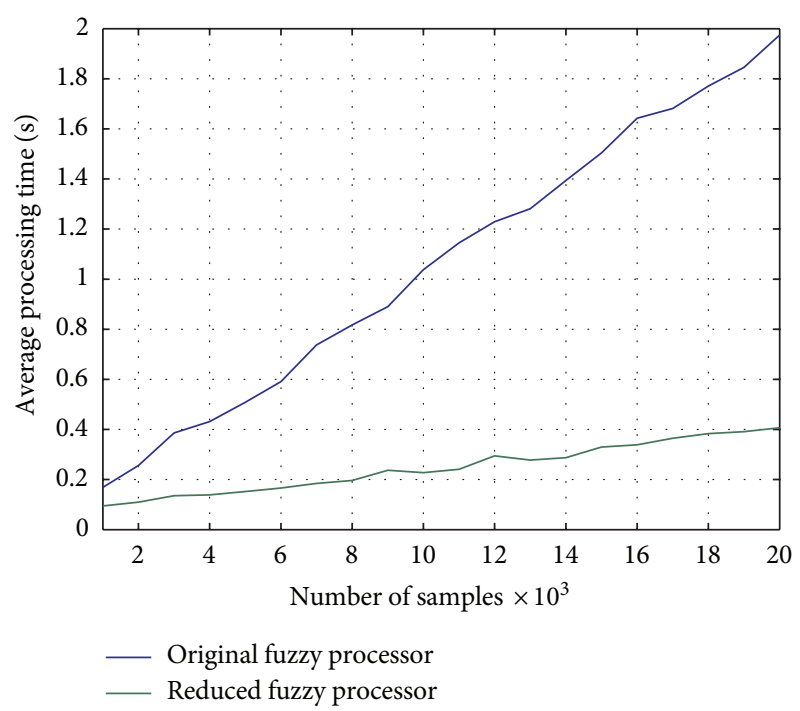

FIGURE 29: Processing time of the original and the reduced fuzzy processors.

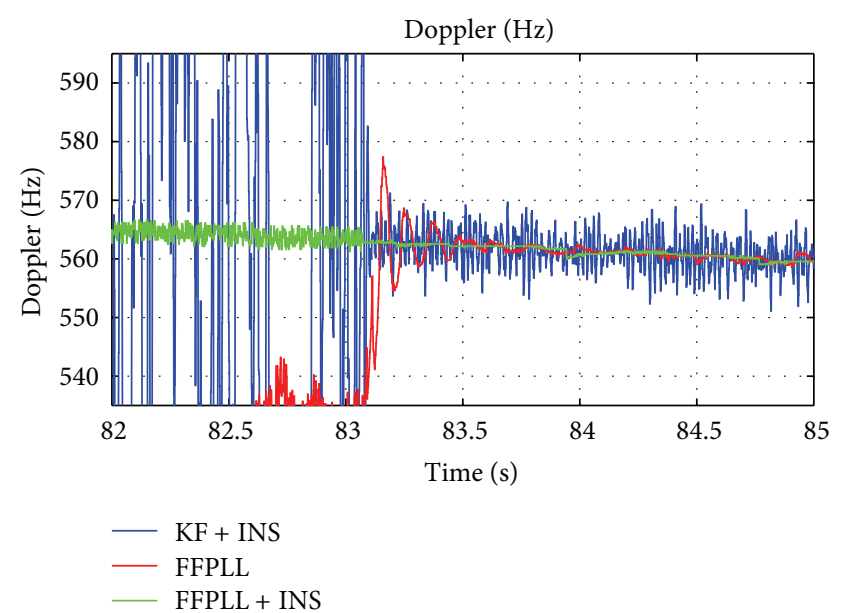

FIGURE 30: Estimated Doppler for PRN 16 using KF + INS, FFPLL, and INS/FFPLL magnified for transient time illustration. 


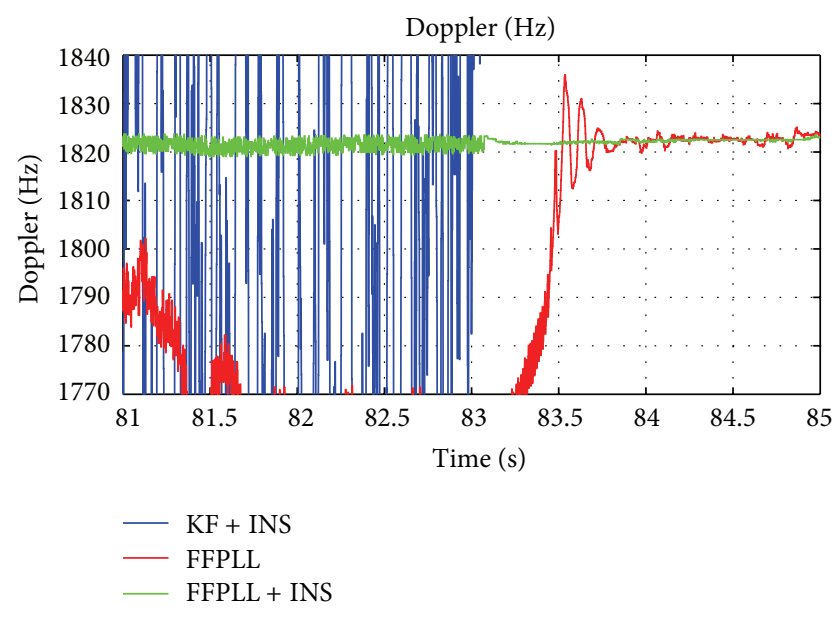

Figure 31: Estimated Doppler for PRN 13 using KF + INS, FFPLL, and FFPLL + INS magnified for transient time illustration.

switch back to tracking mode once jamming ended, unless a new acquisition process was initiated. For this reason, only nonstandard tracking algorithms are shown later in the analysis. However, an example of standard tracking results will first be shown to illustrate the above. Figure 24 shows the estimated Doppler of PRN 13 during part of the scenario. The period started with 55 seconds in static mode followed by an acceleration period and then two jamming periods of a few seconds. Although this type of dynamics is not close to the missile dynamics, features that cannot be assessed with a simulator can be assessed in this case. In this figure, a comparison between the standard PLL $(\mathrm{BW}=18 \mathrm{~Hz})$ and the original FFPLL is shown. Both tracking loop performances are directly compared with the reference INS Doppler.

The FFPLL is able to retrieve the tracking mode, and its estimated Doppler standard deviation, calculated using 10 seconds moving windows, is about 10 times lower than the standard PLL Doppler standard deviation using the same approach as that illustrated in Figure 25. Standard PLL tracking with wide $\mathrm{BW}$ tracking results show that it is not able to reacquire the signal directly after the signal interference period ends. In this case, the receiver has to switch back to acquisition mode, which consumes time.

Figures 26 and 27 show the Doppler calculated for PRN 16 and PRN 13 using KF + INS, FFPLL, and INSassisted FFPLL, respectively. The fuzzy algorithms are able to reacquire signal lock after the jamming period. KF signal reacquisition operation is not consistent because it diverges during jamming periods, and if the erroneously estimated frequency is greater than the pull-in range, it will not be able to reacquire frequency tracking. Therefore, the model used requires more intensive tuning for this specific problem. In addition, it is observed that the estimated Doppler in the Kalman filter case is diverging to a greater extent than FFPLL during the interference period. In the INS-assisted FFPLL case, the estimated Doppler is only oscillating around the required phase tuning frequency thanks to the Doppler measurements provided by the INS. Furthermore, only nine rules related to the phase input are used, which reduces the calculated processing time, as compared to that of the FFPLL. The computational load measured by the processing time is averaged through the simulated and real data experiments and found to be reduced by a factor of about $30 \%$ between the standalone and the assisted FFPLL, as shown in Figure 28. Because the computation of general Fuzzy Logic control can be complex, it is of particular interest to reduce the processing time with a reduced set of fuzzy rules in the INS-aided FFPLL. The results of individual tests, which were performed to compare the processing times of the original and the reduced fuzzy processors, are shown in Figure 29. The tests were applied for different number of samples, and an average of 5 tries is taken for each number/processor type. This test further confirms that the processing time is drastically reduced thanks to the INS adding data.

Even though the INS-aided FFPLL is not able to maintain phase lock during the jamming period, its Doppler frequency estimate is much accurate than the standalone FFPLL. In addition, the transient time required to retrieve the correct signal measurements is much faster as compared to the standalone FFPLL solution, which is very crucial for missile applications where the target can be missed or the missile can be lost in this short time delay. This conclusion, which is consistent with the tracking results for the 10 satellites signals that were available during the experiment, is illustrated in Figures 30 and 31 for PRN 16 and PRN 13, respectively. Even when the KF is assisted by INS, its divergence during jamming is obvious due to large and unmodeled noise, which was assumed to be following a Gaussian distribution.

\section{Conclusions}

In this paper, a new fuzzy based GPS signal tracking loop is designed and tested. A new approach for Fuzzy Logic-based GPS signal tracking using INS-based Doppler aiding is also presented as a modification of the original fuzzy tracking system.

The system is experimentally tested using simulated and live GPS signals in the presence of intentional jamming. Experimental assessment shows that by using a good quality IMU, as is the case for missiles and fighters, the new INSassisted fuzzy tracking is providing better tracking performance as compared to standard and Kalman filter based tracking loops in terms of robustness. Continuous tracking is provided even during very high dynamics conditions and recovered quickly if tracking is lost due to jamming. More accurate and less noisy Doppler estimates are provided. Because the number of rules is reduced from 81 to 9 when the INS is used to assist the FFPLL, the fuzzy processor computation time is reduced by around $30 \%$. Finally, when the GPS signal is lost during the jamming period, the time required to reacquire the signal is shorter than the original fuzzy and Kalman filter based tracking systems. As a future work and to generalize the obtained results, more simulated scenarios will be tested using the proposed algorithm and under different conditions. 


\section{References}

[1] K. Borre, D. M. Akos, N. Bertelsen, P. Rinder, and S. H. Jensen, A Software-Defined GPS and GALILEO Receiver, a Single Frequency Approach, Birkhäuser, Boston, Mass, USA, 2007.

[2] P. Ward, "Performance comparisons between FLL, PLL and a novel FLL-assisted-PLL carrier tracking loop under RF interference conditions," in Proceedings of the 11th International Technical Meeting of the Satellite Division of the Institute of Navigation, pp. 783-795, Nashville, Tenn, USA, 1998.

[3] R. E. Best, Phase-Locked Loops: Design, Simulation, and Applications, McGraw-Hill, New York, NY, USA, 4th edition, 1999.

[4] E. D. Kaplan and C. J. Hegarty, Understanding GPS: Principles and Applications, Artech House, Norwood, Mass, USA, 2nd edition, 2006.

[5] C. O'Driscoll, D. Borio, M. Petovello, T. Williams, and G. Lachapelle, "The soft approach: a recipe for a multi-system, multi-frequency GNSS receiver," InsideGNSS, vol. 4, pp. 46-51, 2009.

[6] R. G. Brown and P. Y. C. Hwang, Introduction to Random Signals and Applied Kalman Filtering with Matlab Exercises and Solutions, John Wiley \& Sons, New York, NY, USA, 3rd edition, 1997.

[7] T. J. Ross, J. M. Booker, and W. J. Parkinson, Fuzzy Logic and Probability Applications, American Statistical Association Society for Industrial and Applied Mathematics, Alexandria, Va, USA, 2002.

[8] A. M. Kamel, "Design and testing of an intelligent GPS tracking loop for noise reduction and high dynamics applications," in Proceedings of the 23rd International Technical Meeting of the Satellite Division of the Institute of Navigation (ION GNSS'10), pp. 3235-3243, Portland, Ore, USA, September 2010.

[9] A. M. Kamel, D. Borio, J. Nielsen, and G. Lachapelle, "Mitigation for missiles (fuzzy logic and intelligent tracking loops cope with interference)," GPS World, 2011, http://www .gpsworld.com/defense/precision-guidance/mitigation-missiles-11710.

[10] R. E. Phillips and G. T. Schmidt, "GPS/INS integration," Neuilly sur Seine, France, 1996.

[11] D. Simon and H. El-Sherief, "Fuzzy phase-locked loops," in Proceedings of the IEEE Position Location and Navigation Symposium, pp. 252-259, Las Vegas, Nev, USA, April 1994.

[12] A. Hiliuta, R. Landry, and F. Gagnon, "Fuzzy corrections in a GPS/INS hybrid navigation system," IEEE Transactions on Aerospace and Electronic Systems, vol. 40, no. 2, pp. 591-600, 2004.

[13] S. Alban, D. Akos, and S. M. Rock, "Performance analysis and architectures for INS-aided GPS tracking loops," in Proceedings of ION National Technical Meeting of the Institute of Navigation (NTM), Fairfax, Va, USA, 2003.

[14] J. D. Gautier and B. W. Parkinson, "Using the GPS/INS generalized evaluation tool (GIGET) for the comparison of loosely coupled, tightly coupled and ultra-tightly coupled integrated navigation systems," in Proceedings of ION 59th Annual Meeting/CIGTF 22nd Guidance Test Symposium, Albuquerque, NM, USA, 2003.

[15] M. G. Petovello and G. Lachapelle, "Comparison of vectorbased software receiver implementations with application to ultra-tight GPS/INS integration," in Proceedings of the 19th International Technical Meeting of the Satellite Division of the Institute of Navigation (ION GNSS'06), pp. 1790-1799, Fort Worth Tex, USA, September 2006.
[16] R. Babu, J. Wang, and G. Rao, "Analysis of ultra-tight GPS/INS integrated system for navigation performance," in Proceedings of the International Conference on Signal Processing Communications and Networking (ICSCN'08), pp. 234-237, Chennai, India, January 2008.

[17] M. G. Petovello, C. O. 'Driscoll, and G. Lachapelle, "Ultra-tight GPS/INS for carrier phase positioning in weak signal environments," in Proceedings of the NATO RTO SET-104 Symposium on Military Capabilities Enabled by Advances in Navigation Sensors, Antalya, Turkey, 2007.

[18] G. Chen and T. T. Pham, Fuzzy Sets, Fuzzy Logic, and Fuzzy Control Systems, CRC Press, New York, NY, USA, 2001.

[19] B. Kosko, Neural Networks and Fuzzy Systems: A Dynamical Systems Approach to Machine Intelligence, Prentice Hall, Englewood Cliffs, NJ, USA, 1992.

[20] A. M. M. Kamel, Context aware high dynamics GNSS-INS for interference mitigation [Ph.D. thesis], Geomatics Engineering, University of Calgary, Calgary, Canada, 2011.

[21] A. J. V. Dierendonck, "GPS receivers," in Global Positioning System: Theory and Applications, B. W. Parkinson, Ed., vol. 1, AIAA, Los Altos, Calif, USA, 1996.

[22] P. Crosta and T. Alenia, "A novel approach to the performance evaluation of an arctangent discriminator for phase locked loop and application to the carrier tracking of the ionospheric scintillation," in Proceedings of the ENC-GNSS 2009, Naples, Italy, 2009.

[23] Spirent, Signal Generator Hardware User Manual, Spirent Communications, 2006.

[24] A. M. Kamel, D. Borio, J. Nielsen, and G. Lachapelle, "Interference mitigation for highly dynamic GPS receivers using intelligent tracking loops," in Proceedings of the International Technical Meeting of the Institute of Navigation (ION ITM'11), pp. 374-383, San Diego, Calif, USA, January 2011.

[25] J. Farrell, Aided Navigation: GPS with High Rate Sensors, vol. 1, McGraw-Hill, New York, NY, USA, 1st edition, 2008.

[26] G. M. Siouris, Missile Guidance and Control Systems, Springer, New York, NY, USA, 2004.

[27] J. Wendel and G. F. Trommer, "Tightly coupled GPS/INS integration for missile applications," Aerospace Science and Technology, vol. 8, no. 7, pp. 627-634, 2004.

[28] P. D. Groves, Principles of GNSS, Inertial, and Multisensor Integrated Navigation Systems, Artech House, Norwood, Mass, USA, 2008

[29] D. E. Lewis, "Ultra-tightly coupled GPS/INS tracking performance," in Proceedings of the AIAA's 3rd Annual Aviation Technology, Integration, and Operations (ATIO'03), Tech Denver, Colo, USA, 2003.

[30] N. Elsheimy, ENGO 623 Lecture Notes: Inertial Techniques and INS/DGPS Integration, Department of Geomatics Engineering, University of Calgary, Calgary, Canada, 2007.

[31] W. Pedrycz and F. Gomide, An Introduction to Fuzzy Sets: Analysis and Design, MIT Press, Cambridge, Mass, USA, 1998.

[32] P. Closas, C. Fernandez-Prades, J. Diez, and D. de Castro, "Nonlinear Bayesian tracking loops for multipath mitigation," International Journal of Navigation and Observation, vol. 2012, article 15, 2012.

[33] Honeywell, HG1700 Inertial measurement unit, 2006, http:// www.honeywell.com/sites/servlet/com.merx.npoint.servlets .DocumentServlet?docid=D7B652202-0601-F56B-1B7F829F1A7109E4. 
[34] NovAtel, Inertial explorer data sheet, 2011, http://www.novatel.com/assets/Documents/Waypoint/InertialExplorer.pdf.

[35] GlobalSources, "Leica TCR705 reflectorless [TCR705] product details," 2011.

[36] A. H. Jazwinski, Stochastic Processes and Filtering Theory, vol. 64, Academic Press, New York, NY, USA, 1970.

[37] J. Kouba and P. Héroux, "Precise point positioning using IGS orbit and clock products," GPS Solutions, vol. 5, pp. 12-28, 2001.

[38] M. L. Psiaki, "Smoother-based GPS signal tracking in a software receiver," in Proceedings of the ION GPS 2001, Salt Lake City, Utah, USA, 2001.

[39] M. L. Psiaki, T. E. Humphreys, A. P. Cerruti, S. P. Powell, and P. M. Kintner, "Tracking L1 C/A and L2C signals through ionospheric scintillations," in Proceedings of the 20th International Technical Meeting of the Satellite Division of the Institute of Navigation (ION GNSS'07), pp. 246-268, Fort Worth, Tex, USA, September 2007. 

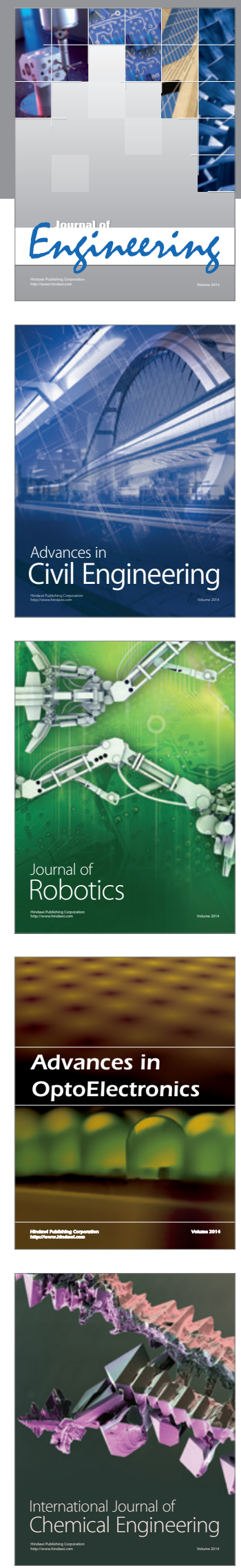

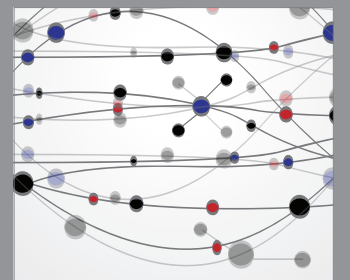

The Scientific World Journal
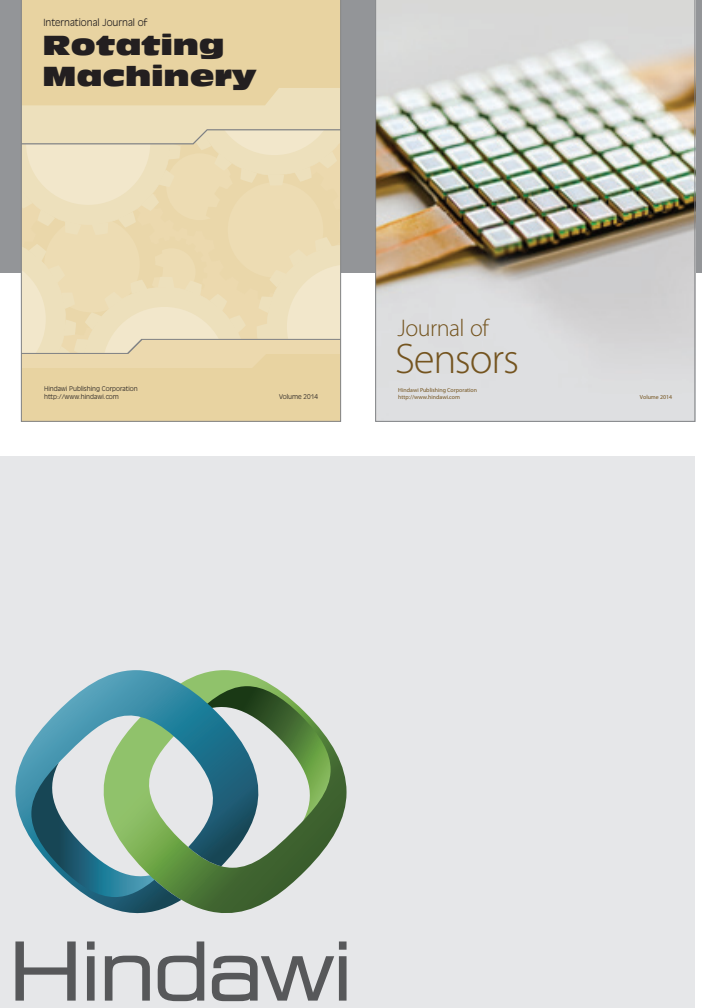

Submit your manuscripts at http://www.hindawi.com
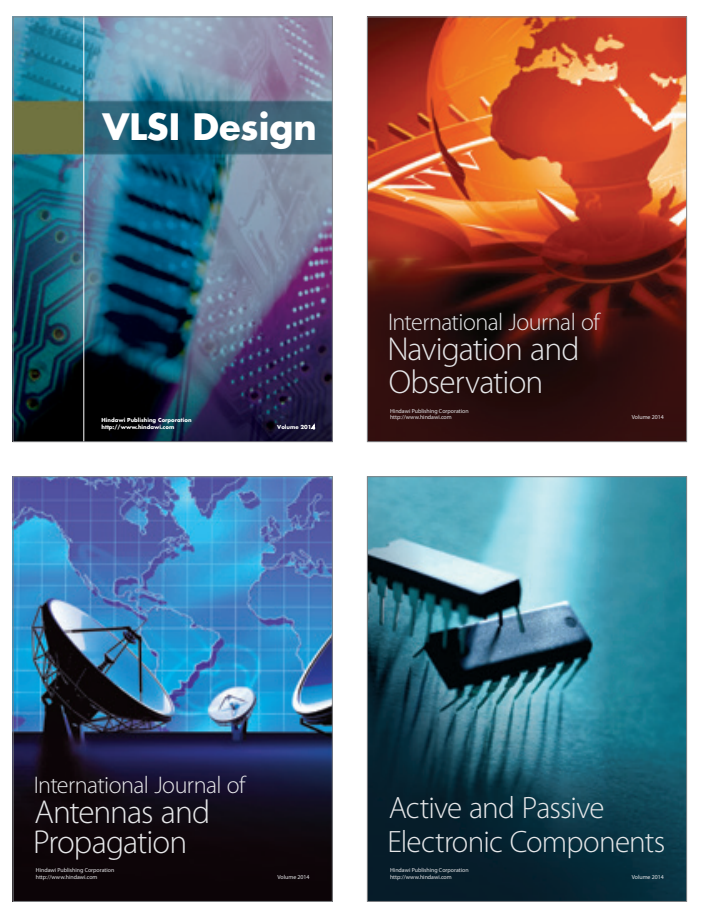
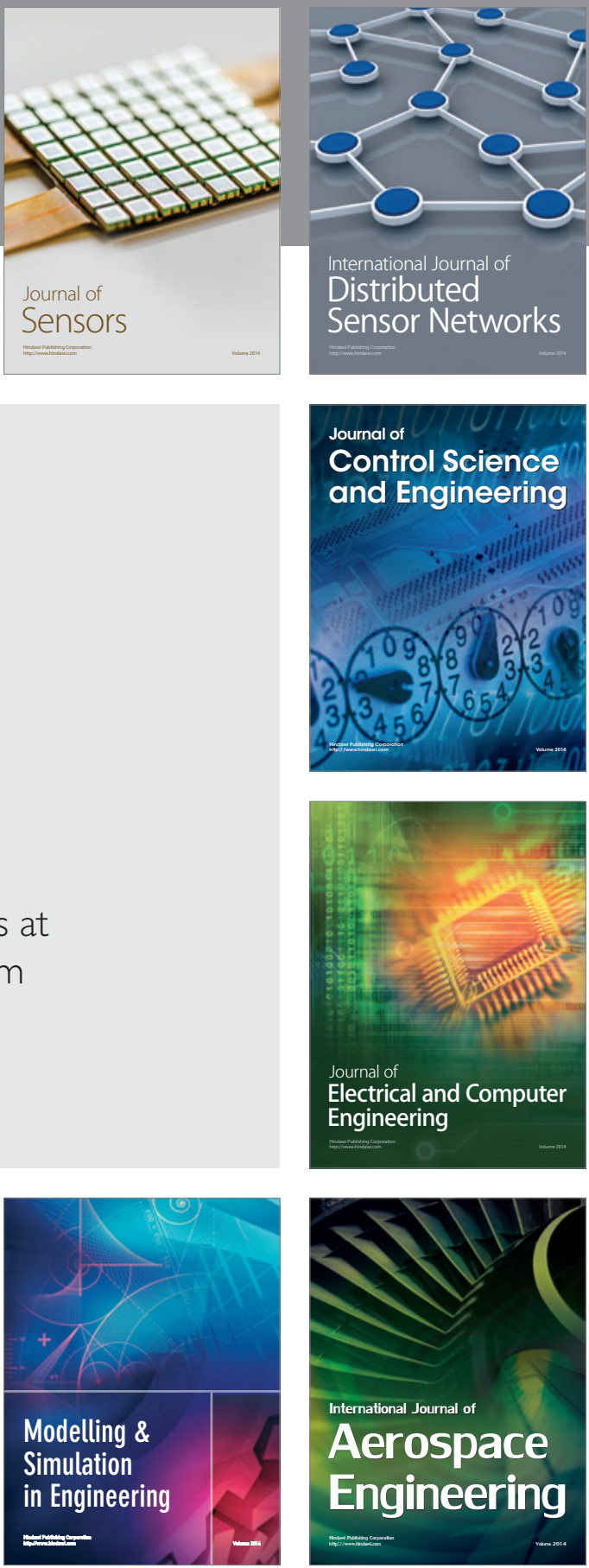

Journal of

Control Science

and Engineering
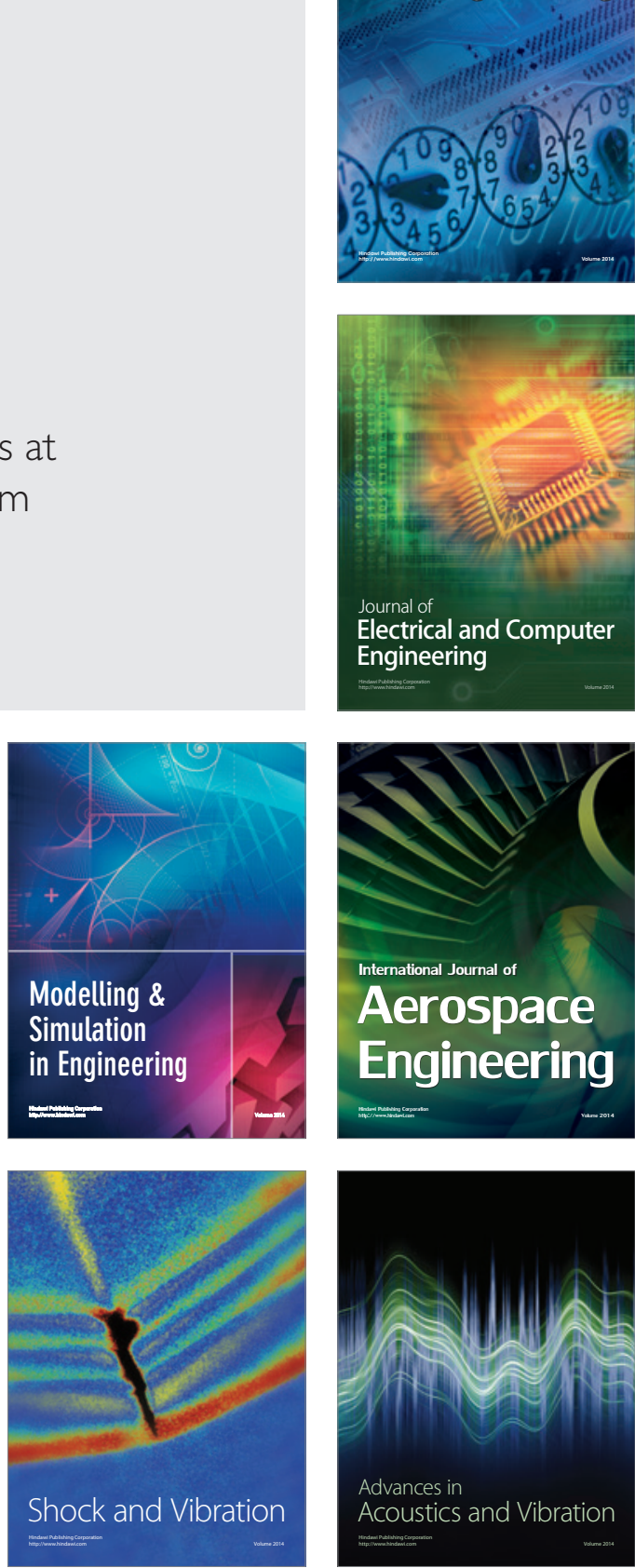